ARTICLE

https://doi.org/10.1038/s41467-019-09728-3

\title{
Origin and differentiation trajectories of fibroblastic reticular cells in the splenic white pulp
}

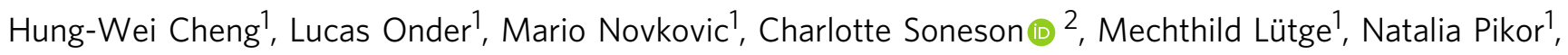
Elke Scandella ${ }^{1}$, Mark D. Robinson (1) ${ }^{2}$, Jun-ichi Miyazaki ${ }^{3}$, Anne Tersteegen (1) ${ }^{4}$, Ursula Sorg ${ }^{4}$, Klaus Pfeffer ${ }^{4}$, Thomas Rülicke ${ }^{5}$, Thomas Hehlgans ${ }^{6}$ \& Burkhard Ludewig (i) ${ }^{1}$

The splenic white pulp is underpinned by poorly characterized stromal cells that demarcate distinct immune cell microenvironments. Here we establish fibroblastic reticular cell (FRC)specific fate-mapping in mice to define their embryonic origin and differentiation trajectories. Our data show that all reticular cell subsets descend from multipotent progenitors emerging at embryonic day 19.5 from periarterial progenitors. Commitment of FRC progenitors is concluded during the first week of postnatal life through occupation of niches along developing central arterioles. Single cell transcriptomic analysis facilitated deconvolution of FRC differentiation trajectories and indicated that perivascular reticular cells function both as adult lymphoid organizer cells and mural cell progenitors. The lymphotoxin- $\beta$ receptor-independent sustenance of postnatal progenitor stemness unveils that systemic immune surveillance in the splenic white pulp is governed through subset specification of reticular cells from a multipotent periarterial progenitor cell. In sum, the finding that discrete signaling events in perivascular niches determine the differentiation trajectories of reticular cell networks explains the development of distinct microenvironmental niches in secondary and tertiary lymphoid tissues that are crucial for the induction and regulation of innate and adaptive immune processes.

\footnotetext{
${ }^{1}$ Institute of Immunobiology, Kantonsspital St. Gallen, 9007 St. Gallen, Switzerland. ${ }^{2}$ Institute of Molecular Life Sciences and SIB Swiss Institute of Bioinformatics, University of Zurich, 8057 Zurich, Switzerland. ${ }^{3}$ Division of Stem Cell Regulation Research, Osaka University Medical School, 565-0871 Osaka, Japan. ${ }^{4}$ Institute of Medical Microbiology and Hospital Hygiene, University of Düsseldorf, 40225 Düsseldorf, Germany. ${ }^{5}$ Institute of Laboratory Animal Science, University of Veterinary Medicine Vienna, 1210 Vienna, Austria. ${ }^{6}$ Institute of Immunology, Regensburg Center for Interventional Immunology (RCl) and University Medical Center of Regensburg, 93053 Regensburg, Germany. Correspondence and requests for materials should be addressed to B.L. (email: burkhard.ludewig@kssg.ch)
} 


\section{T} he mammalian spleen is the largest secondary lymphoid organ (SLO) that contains specialized microenvironments for hematopoiesis and control of erythrocyte turnover in the red pulp, while immune protection against blood-borne pathogens is secured in the white pulp $p^{1,2}$. Asplenic individuals and patients undergoing splenectomy bear a life-long risk of overwhelming infections ${ }^{3}$ and are predisposed to succumb to septic shock ${ }^{4}$. The first line of immune defense is provided by the marginal zone of the white pulp where microbial antigens are captured by myeloid cells 5,6 and innate immune responses are initiated ${ }^{7}$. Both T- and B-cell compartments of the white pulp are underpinned by specialized fibroblastic stromal cells that provide a physical scaffold and generate chemokines and cytokines to facilitate efficient interaction between immune cells ${ }^{8,9}$. Selective loss of white pulp reticular cells, for example during viral infection, precipitates profound immunodeficiency ${ }^{10}$ and immune functionality is restored only once reticular cell networks have been rebuilt ${ }^{11}$.

Generation of functional immune environments in lymph nodes depends on maturation and functional specialization of fibroblastic reticular cells (FRC) that are associated with the broad expression of the mucin-type transmembrane protein podoplanin $(\mathrm{PDPN})^{12-14}$. In the spleen, however, PDPN expression is expressed mainly by T-cell zone reticular cells (TRC), which produce the homeostatic chemokines CCL19 and CCL2 $1^{15}$. Splenic B-cell zone reticular cells, as their counterparts in lymph nodes, express the chemokine CXCL13 15,16 and encompass the follicular dendritic cell (FDC) fraction, which retain antigen on their surface through the expression of complement receptors ${ }^{17}$. The marginal zone in human and murine spleens is underpinned by marginal reticular cells (MRC), which express the adhesion molecule MAdCAM-1 and CXCL13 ${ }^{18,19}$, and thereby foster interaction with innate lymphoid cells and B cells during the initiation of antibody responses ${ }^{19}$. Interestingly, while lymph node reticular cell subsets have been characterized extensively ${ }^{20,21}$, molecular details on splenic white pulp reticular cell lineages have remained elusive.

The analysis of cellular lineage relationship requires knowledge on the embryonic origin and subsequent commitment steps that determine the critical nodes in the differentiation trajectories. Both red and white pulp fibroblastic stromal cells descend from stem cells in the embryonic splenopancreatic mesenchyme ${ }^{2}$. Early Nkx2-5 and Islet1-positive mesenchymal progenitors that appear at embryonic day (E) 10.5 have been shown to participate in the generation of white pulp reticular cell subsets ${ }^{18}$. Notably, splenic $\mathrm{Nkx} 2-5^{+}$Islet $1^{+}$progenitors contribute as well to the fibroblastic stromal cell pool in the red pulp ${ }^{18}$. Moreover, the finding that cardiac $\mathrm{Nkx} 2-5^{+}$progenitors give rise to both smooth muscle cells and cardiomyocytes ${ }^{22}$ indicates that Nkx2-5 expression occurs as one of the first differentiation steps of various mesenchymal cell populations. Hence, it is conceivable that the critical process of white pulp reticular cell progenitor commitment ensues during later stages of spleen development and that these cells may function as the main drivers for the structural organization of the white pulp.

Since the nature and the habitat of committed splenic white pulp mesenchymal lymphoid tissue organizer (mLTo) cells during embryonic development and in the adult have remained largely unknown, we combine single-cell genetic and molecular approaches to record the differentiation trajectories of white pulp reticular cells. The combination of in vivo cell-fate mapping and single-cell RNA-seq (scRNA-seq) analysis reveals that differentiation of white pulp reticular cell networks is dependent on lymphotoxin- $\beta$ receptor (LT $\beta$ R) signaling, while mural cell specification and sustenance of multipotent adult progenitor cells follow LT $\beta$ R-independent trajectories. The close linkage of
FRC and mural cell development suggests that distinct microenvironmental niches in secondary and tertiary lymphoid organs develop in a hierarchical process with LT $\beta$ R signaling serving as a main switch and other-only partially known-secondary signals driving FRC subset specification.

\section{Results}

Reticular cell subsets in the splenic white pulp. The $\mathrm{Ccl19}$ promoter is well-suited to genetically target the main subsets of FRC in lymph nodes ${ }^{13,16,23,24}$ and Peyer's patches ${ }^{25}$. In the spleen, the Ccl19-Cre transgene highlights fibroblastic stromal cells in the T- and B-cell zones of the white pulp (Supplementary Fig. 1a) highlighting T-cell zone reticular cells expressing CCL21 (Supplementary Fig. 1b) and CXCL13-producing reticular cells in the B-cell zone (Supplementary Fig. 1c). Since constitutive Cre recombinase expression is not suitable to pinpoint cellular progenitor-progeny relationship, we generated an inducible cellfate mapping system based on the expression of the tetracyclin transactivator (tTA) in Ccl19-expressing cells. The resulting Ccl19-tTA strain was crossed with LC-126 and R26R-EYFP mice to permit timely regulated Cre expression in the triple-transgenic mouse model termed Ccl19-iEYFP (Fig. 1a). In the spleen, the Ccl19-iEYFP transgene targeted fibroblastic stromal cells throughout the white pulp (Fig. 1b and Supplementary Fig. 1d), recapitulating the phenotype of the Ccl19-Cre mouse model (Supplementary Fig. 1a-c). Moreover, the expression of the redfluorescent protein tdTomato (tdTom) monitors current $\mathrm{Ccl} 19$ promoter activity in the T-cell zone and the marginal zone (Fig. 1c and Supplementary Fig. 1d). Pre- and postnatal exposure to doxycycline (Dox) completely blocked Cre recombinasedependent EYFP expression, while production of the tdTom was not affected (Supplementary Fig. 1e) indicating that Cre recombinase activity can be stringently regulated in this model system. High-resolution confocal microscopy facilitated the distinction of the main $\mathrm{EYFP}^{+}$reticular cell subsets with MRC in the marginal zone expressing MAdCAM-1 and CD157, FDC in the B-cell follicle that exhibit complement receptor 1 and 2 (CD35 and CD21, respectively) and CD157 expression, TRC with PDPN and aSMA expression and stem cell antigen-1 (Sca-1)-positive cells forming a perivascular reticular cell (PRC) network (Fig. 1d).

Expression of the LT $\beta \mathrm{R}$ in splenic reticular cells is essential for the formation of the splenic white pulp ${ }^{27,28}$. As expected, cell type-specific $L t b r$-deficiency in Ccl19-iEYFP $L t b r \mathrm{fl} / \mathrm{fl}$ mice led to the failure to form the white pulp with the loss of specific white pulp microenvironments (Fig. 1e). Interestingly, $\mathrm{EYFP}^{+}$cells were still detectable in spleens of conditionally $L t b r$-deficient mice and were identified as aSMA-expressing myofibroblasts surrounding the splenic arterioles (Fig. 1f), suggesting that splenic white pulp formation is abolished when periarterial myofibroblasts fail to respond to lymphotoxin. Next, we used flow cytometric analysis to quantify the effect of cell type-specific $L t b r$ deficiency on white pulp reticular cell development. EYFP ${ }^{+}$ reticular cells constituted only a minor fraction of all nonendothelial splenic stromal cells, which could be separated in $\mathrm{PDPN}^{+}$TRC, $\mathrm{CD} 35^{+}$FDC, and MAdCAM-1 ${ }^{+}$MRC (Fig. 1g and Supplementary Fig. 1f). Moreover, Sca- $1^{+}$cells co-expressed PDGFRa (Fig. 1g), a trait that is characteristic for mesenchymal progenitor cells in the bone marrow ${ }^{29-31}$. Since $\mathrm{CD} 157$ was expressed by TRC, MRC, and FDC (Fig. 1g), co-staining for PDPN and CD157 was used to distinguish Sca-1 ${ }^{+}$PRC from TRC/MRC/FDC subsets leaving a fraction that is termed here as triple-negative cells (TNC) (Fig. 1g). Strikingly, the lack of $L t b r$ expression in $\mathrm{EYFP}^{+}$cells led to an almost complete loss of MRC, TRC and FDC, while the proportion of PRC was significantly elevated (Fig. 1g, h). The fraction of TNC was not affected by the 
a

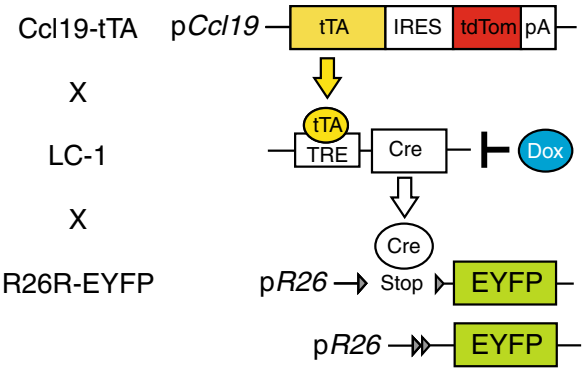

d



EYFP CD4 B220



EYFP CD21/35 CD157
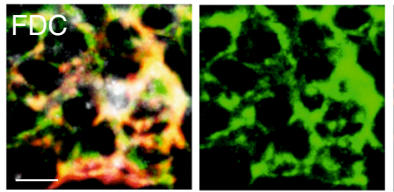

g
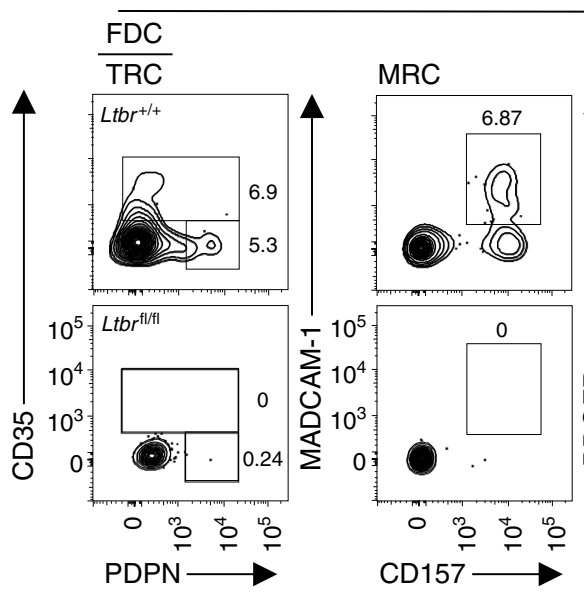

Ccl19-iEYFP
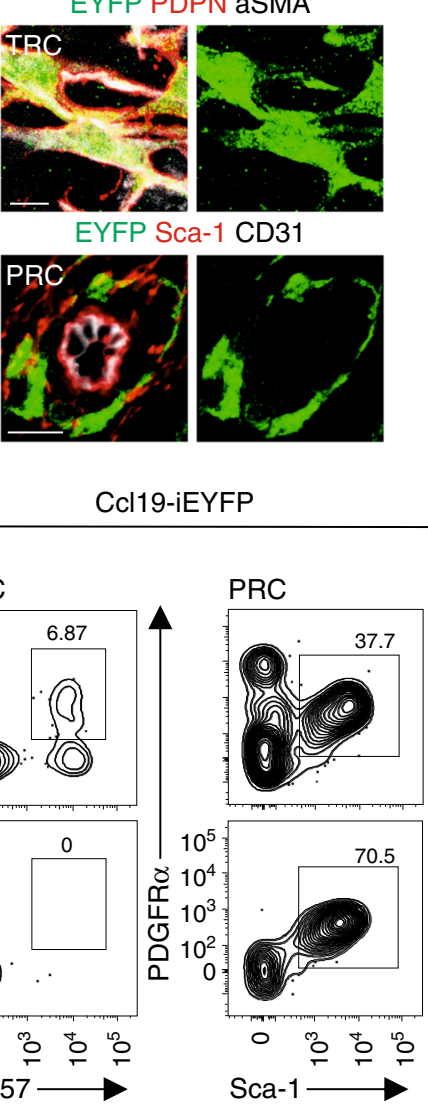

cell type-specific lack of $L t b r$ expression (Fig. $1 \mathrm{~g}, \mathrm{~h}$ ). In sum, these results indicate that mLTo cells are specifically targeted in the Ccl19-iEYFP transgenic mice and suggest that the LT $\beta R$ dependent emergence of distinct reticular cell subsets facilitates the organization of the splenic white pulp.

Defining the mLTo cell habitat in the embryonic spleen. Timed pregnancy and assessment of transgene activity at different embryonic stages in Ccl19-iEYFP mice revealed that aSMA-

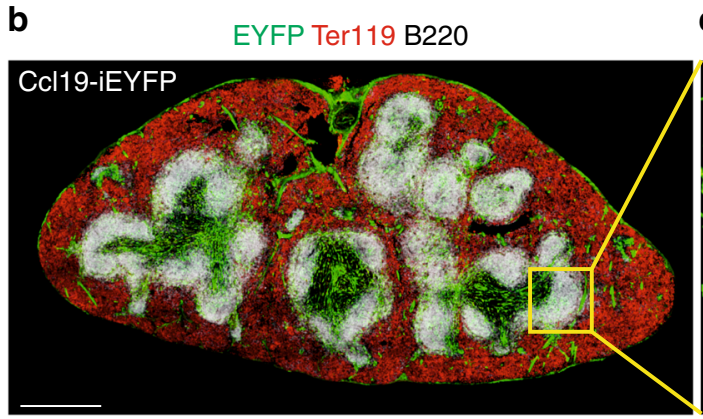

C EYFP tdTom CCL19

e
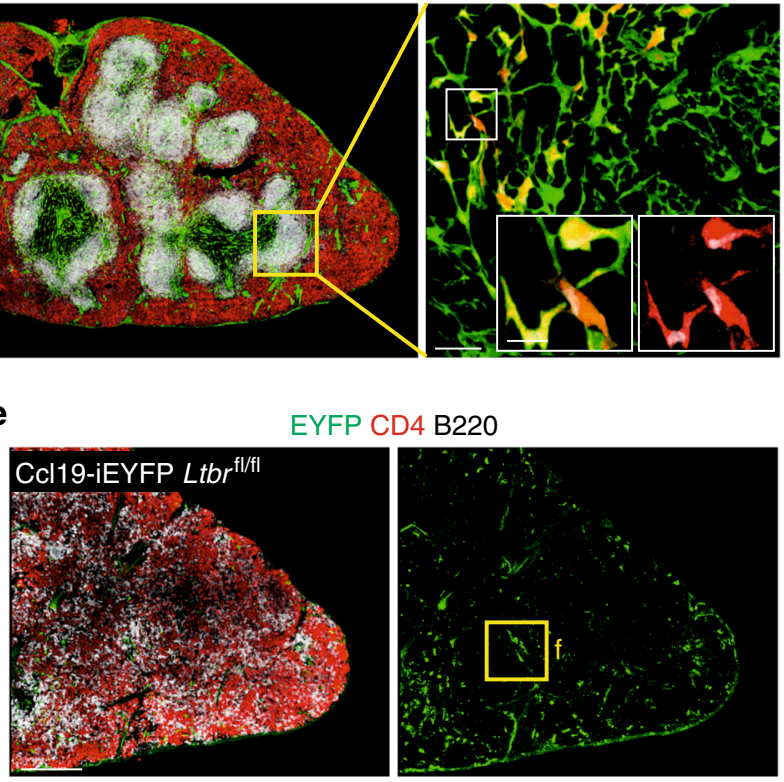

f


h

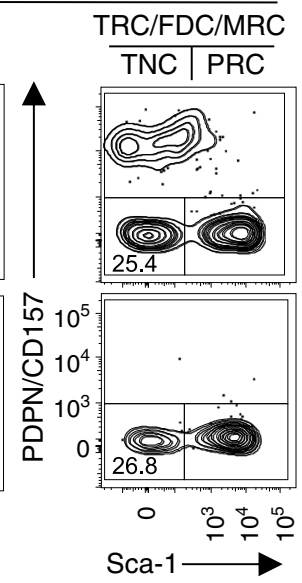

expressing myofibroblasts surrounding the splenic artery started to express the transgene at E19.5 concomitantly with the immigration of $\mathrm{B}$ and $\mathrm{T}$ cells to the fetal spleen (Fig. 2a and Supplementary Fig. 2a and b). At the neonatal stage (P0), expanded peri-arteriolar lymphocytic cuffs were underpinned by $\mathrm{EYFP}^{+}$ cells (Fig. 2a and Supplementary Fig. 2a and b). In a first differentiation step, MAdCAM-1 ${ }^{+}$MRC appeared at postnatal day (P7), forming a primordial marginal zone (Supplementary Fig. 2c). The reticular cell differentiation markers PDPN and 
Fig. 1 Differentiation of splenic white pulp reticular cell populations. a Schematic depiction of the triple transgenic Ccl19-iEYFP mouse model. b Spleen cross-sections from adult Ccl19-iEYFP mice without doxycycline treatment analyzed by confocal microscopy after staining with the indicated antibodies (scale bar $=500 \mu \mathrm{m}$ ). Boxed areas in (b) indicate the splenic white pulp EYFP+ reticular cells analyzed by confocal microscopy after staining with the indicated antibodies in (c, d). Boxed areas in (c) show region of higher magnification in the images (Scale bar $=50 \mu \mathrm{m}$ and $10 \mu \mathrm{m}$ in the boxes). Boxed regions in (d) indicate marginal zone reticular cells (MRC), follicular dendritic cells (FDC), T-cell zone reticular cells (TRC), and perivascular reticular cells

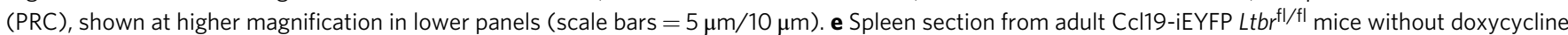
treatment stained with the indicated antibodies and analyzed by confocal microscopy. Box indicates magnified areas in (f) (scale bar in (e) $=200 \mu \mathrm{m}$, scale bars in $(\mathbf{f})=20 \mu \mathrm{m})$. $\mathbf{g}$ Representative flow cytometric analysis of viable CD45-depleted cells from Ccl19-iEYFP and Ccl19-iEYFP Ltbr fl/fl spleens without doxycycline treatment, gated on CD45-TER119-CD31-EYFP+ cells and analyzed for the indicated markers. Values indicate percentage of the respective population. $\mathbf{h}$ Percentage of EYFP + cells from spleens of the indicated mouse strains, based on gating in ( $\mathbf{g}$ ) ( $n=7-8$ mice per group from 2 to 3

independent experiments, mean \pm SEM). Statistical analysis was performed using Mann-Whitney test for (h). Microscopy data are representative for two or more independent experiments ( $n=6$ mice). Source data are provided as Source Data file

CD21/35 became detectable at postnatal week 2 with the beginning of T-cell and B-cell zone segregation (Fig. 2b and Supplementary Fig. 2d). Multicolor flow cytometric analysis revealed that the majority of the perivascular myofibroblasts detected at E19.5 and P0 exhibit a PRC phenotype, while the proportion of reticular cells expressing the differentiation markers PDPN and CD157 (Fig. 2c, d) increased with the growth of the splenic white pulp until week 6 (Supplementary Fig. 2e). Moreover, assessment of Ki67 expression revealed that the expansion of the white pulp was accompanied by high-rate proliferation of $\mathrm{EYFP}^{+}$cells (Fig. 2e, f), suggesting that splenic white pulp formation is linked to distinct proliferation and differentiation processes of reticular cells that are triggered by the commitment of periarterial myofibroblasts at E19.5. Moreover, these data resolve that cells targeted by the Ccl19-tTA transgene function as embryonic mLTo cells during white pulp development.

Tracking the progeny of embryonic mLTo cells. To assess whether embryonic mLTo cells exhibit multipotent differentiation potential, we permanently tagged the first transgene-positive mLTo cells by treating pregnant Ccl19-iEYFP and Ccl19-iEYFP $L t b r^{\mathrm{f} / \mathrm{fl}}$ dams at E19.5 with Dox and provided the compound to the lactating dam, and after weaning to the offspring, via drinking water up until week 6 (Fig. 3a). Both E19.5-6 week fate-mapped Ltbr-proficient (Fig. 3b) and Ltbr-deficient (Fig. 3c) reticular cells were found mainly in areas surrounding the main branch of the splenic artery. $L t b r$-proficiency facilitated differentiation of embryonic mLTo cells into PDPN ${ }^{+}$TRC (Fig. 3d), MAdCAM- $1^{+}$ MRC (Fig. 3d) and CD21/35 ${ }^{+}$FDC (Fig. 3e). Moreover, fatemapped cells exhibited additional functional traits of FRC subsets such as CCL19 expression in TRC and CXCL13 expression in FDC (Supplementary Fig. 3a, b). In contrast, neither of the specialized reticular cell subsets appeared in spleens of fatemapped Ccl19-iEYFP $L t b r^{\mathrm{fl} / \mathrm{fl}}$ mice where $L t b r$-deficient $\mathrm{EYFP}^{+}$ cells remained as aSMA-expressing myofibroblasts in their periarterial niches (Fig. 3f, g). Flow cytometric analysis confirmed the differentiation block in Ltbr-deficient fate-mapped mLTo cells (Fig. 3h and Supplementary Fig. 3c) and showed that Ltbrdependent arrest of reticular cell development resulted in an accumulation of PRC (Fig. $3 \mathrm{i}$ and Supplementary Fig. 3c). Interestingly, the proliferation rates of $\mathrm{EYFP}^{+}$fate-mapped cells determined as fraction of $\mathrm{Ki}^{+}{ }^{+}$cells were not affected by the Ltbr ablation (Fig. 3j, k). Taken together, these data reveal that embryonic mLTo cells operate as multipotent reticular cell progenitors and indicate that the maintenance of the PRC network is independent of LT $\beta R$ signaling.

To further investigate the progenitor-progeny relationship of embryonic mLTo cells and FRC subsets at the single-cell level, we crossed the Ccl19-tTA mouse strain to the LC-1 strain and the multicolor reporter Brainbow2.1 mouse line (Ccl19-iBrainbow 2.1$)^{32}$. As expected, FRC were labeled randomly by different fluorescent proteins in the absence of doxycycline application (Supplementary Fig. 3d-e). In contrast, individual cell fatemapping of E19.5 mLTo cells to 6 weeks revealed that single colored $\mathrm{EYFP}^{+}$or $\mathrm{RFP}^{+}$cell clusters contain distinct FRC subsets with $\mathrm{PDPN}^{+}$TRC, $\mathrm{CD} 21 / 35^{+} \mathrm{FDC}$ or single MAdCAM1 ${ }^{+} \mathrm{MRC}$, which is indicative of locally proliferating progenitors (Supplementary Fig. $3 \mathrm{f}-\mathrm{h}$ ). These results suggest that FRC subset specification from embryonic mLTo cells occurs through clonal expansion in local microenvironments during the interaction with hematopoietic cells.

Consecutive mLTo cell commitment along the arteriolar tree. The finding that progeny of E19.5 mLTo cells congregate mainly in the area surrounding the main splenic artery (Fig. 3b) suggested that the process of mLTo cell commitment starts at the central blood supply of the developing organ. Indeed, wholemount scanning of Ccl19-iEYFP embryonic spleens revealed that arterial smooth muscle cells undergo a decisive commitment step at E19.5 along the main branch of the splenic artery (Supplementary Movie 1). Moreover, the finding that the white pulp structures developed in E19.5-6 week fate-mapped Ltbr-deficient mice (Fig. 3c) suggested that Ltbr-proficient mLTo cells could be committed after E19.5. To determine the spatio-temporal parameters underlying mLTo commitment during spleen development, we blocked Cre recombinase activity in Ccl19-iEYFP mice through Dox administration from E19.5 (Fig. 4a) or from P7 (Fig. 4b) to week 6 and compared the expansion of $\mathrm{EYFP}^{+}$ progeny to untreated mice. We found that mLTo cell progeny in distal regions were present at lower density (Fig. 4c), but still contributed to the MRC (Fig. 4c, arrow), TRC (Fig. 4c, arrowhead), and FDC (Fig. 4c, asterisk) subsets. White pulp areas more proximal to the central artery were densely populated with all reticular cell subsets (Fig. 4c). Arresting Cre recombinase activity at P7 was associated with equally dense cell distribution in distal and in proximal (Fig. 4d) areas. Next, we applied a histomorphometric analysis that resolves the concentric distance of $\mathrm{EYFP}^{+}$cells from the central artery at the hilum of the spleen (Fig. 4e). Quantification of the frequency of $\mathrm{EYFP}^{+}$cells in concentric areas relative to the central artery revealed that density and distribution pattern of white pulp reticular cells were determined during the time period of E19.5 to P7 (Fig. 4f). Since fate mapping of potential mLTo cells at E17.5 did not yield any EYFP $^{+}$cells (Supplementary Fig. 3i), we conclude that mLTo cell commitment starts at E19.5 and continues along the growing arteriolar tree during the first week of postnatal growth.

Transcriptome-based subset definition of splenic fibroblasts. To resolve the molecular characteristics of white pulp reticular cells and to determine their differentiation trajectories, we first performed population-based RNA sequencing (popRNA-seq) 


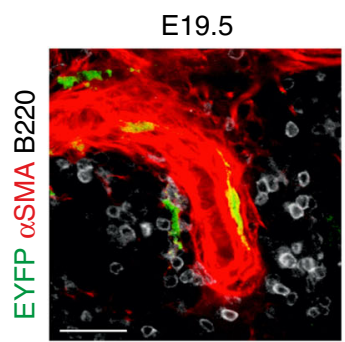

b

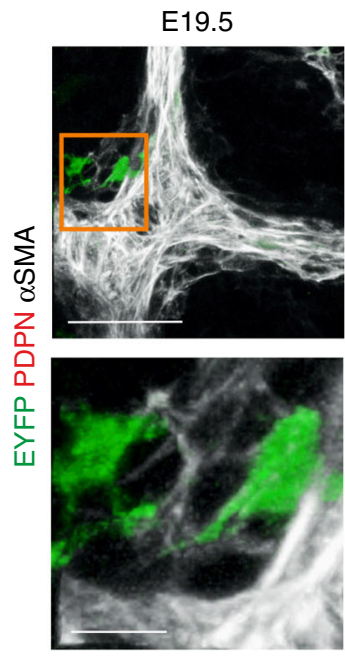

C

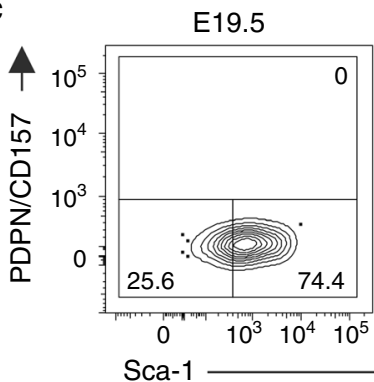

d

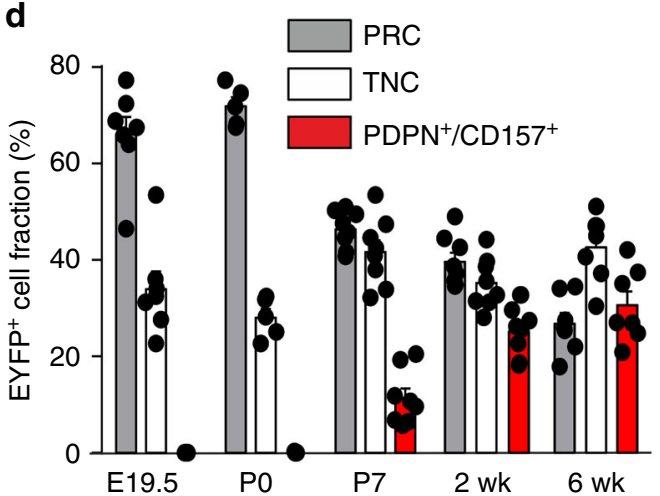

P7

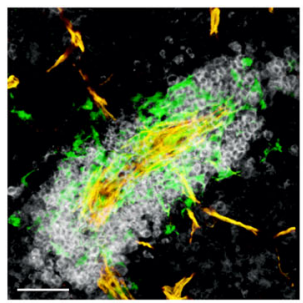

P0

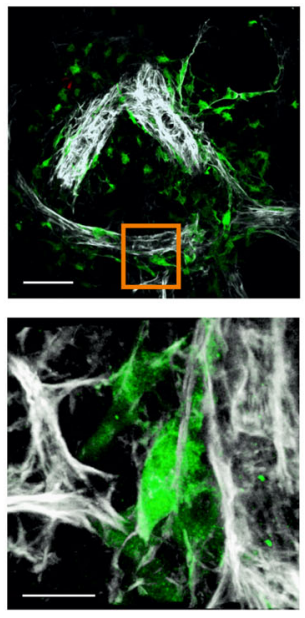

P0

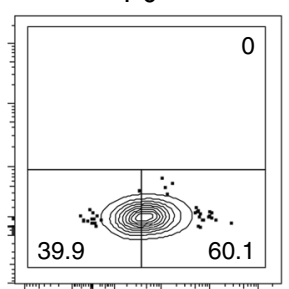

P7

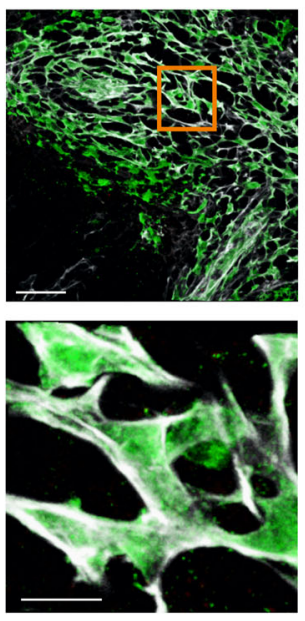

P7

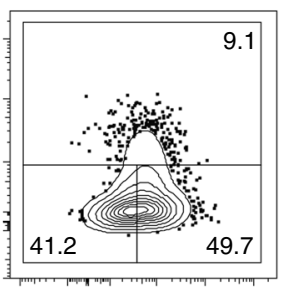

2 wk

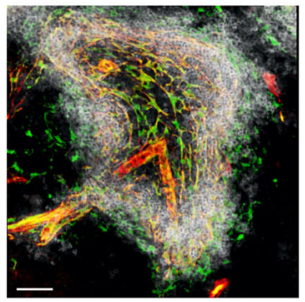

2 wk
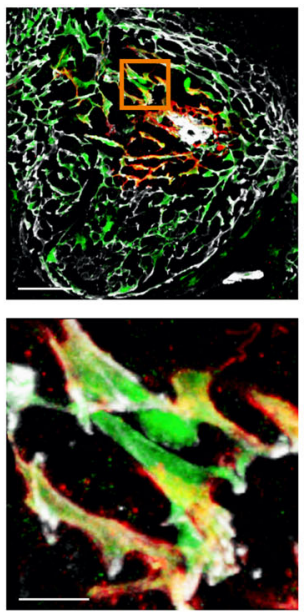

2 wk

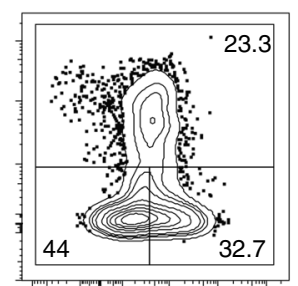

6 wk

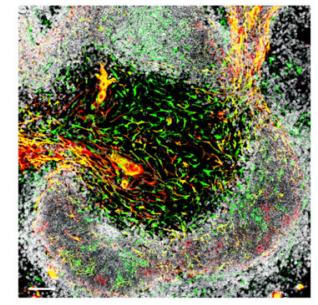

$6 \mathrm{wk}$
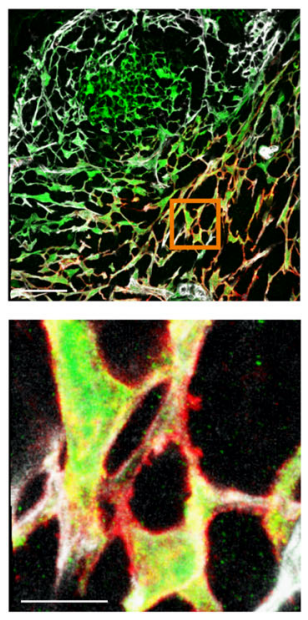

$6 w k$

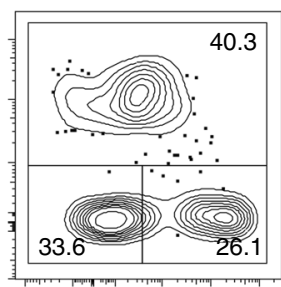

e

EYFP Ki67 $\alpha$ SMA

f

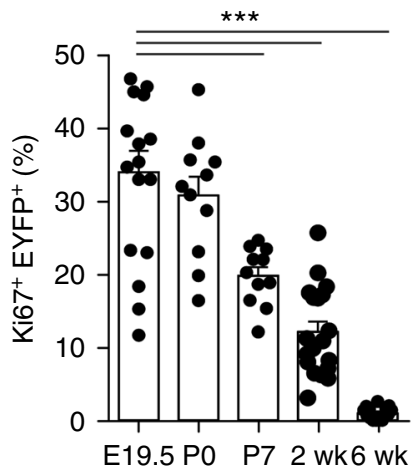

using the established cell marker profiles (Fig. 1g). We analyzed the transcriptome of sorted embryonic mLTo cells based on EYFP expression and adult Ltbr-deficient $\mathrm{EYFP}^{+}$cells to elucidate the molecular basis of postnatal mLTo cell stemness. Data analysis based on differentially expressed genes revealed that the gene expression pattern of embryonic mLTo cells was distinct from adult reticular cells (Supplementary Fig. 4a). Gene set enrichment analysis indicated that embryonic periarterial mLTo cells differ from $L t b r$-arrested adult PRC mainly in terms of antigen processing and presentation and the generation of extracellular matrix components (Supplementary Fig. 4b). Gene-expression analysis provided more detailed molecular insight and revealed that both embryonic mLTo cells and Ltbr-deficient $\mathrm{EYFP}^{+}$ cells expressed transcription factors implicated in spleen 
Fig. 2 Reticular cell subset differentiation during splenic white pulp development. a, b Time course analysis of EYFP-expressing cells in spleens from Ccl19-

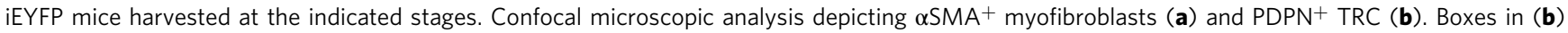
indicate magnified regions in the lower row. Microscopy data are representative for two or more independent experiments ( $n \geq 4$ mice per group). $\mathbf{c}$, $\mathbf{d}$ Flow cytometric analysis of CD45-TER119-EYFP+ cells using antibodies against PDPN, CD157 and Sca-1. Values from one representative experiment indicate percentage of the respective population shown in (c) and cumulated values for proportions of reticular cell subsets shown in (d) ( $n>5$ mice per group from two to three independent experiments, mean \pm SEM). e, $\mathbf{f}$ Assessment of cellular proliferation using Ki67 staining in spleens of Ccl19-iEYFP mice at the indicated age with representative section in (e) and quantification of the proportion of Ki67 expression in EYFP+ cells (f) $(n>4$ mice per group from three independent experiments, mean \pm SEM). Statistical analysis was performed using a one-way ANOVA with Tukey's post test. Source data are provided as Source Data file

organogenesis as well as mesenchymal stem cell markers (Supplementary Fig. 4c). The reduced expression of TRC, MRC, and FDC marker genes in adult $\mathrm{Ltbr}$-deficient $\mathrm{EYFP}^{+}$cells (Supplementary Fig. 4d) suggested that initiation of TRC/MRC/FDC subset specification and concomitant loss of stemness are the major LT $\beta$ R-dependent processes during white pulp formation. Indeed, comparison of popRNA-seq profiles of defined reticular subsets with E19.5 EYFP $^{+}$cells revealed that embryonic mLTo cells were most similar to PRC (Supplementary Fig. 4e). The two cell populations shared mesenchymal stem cell and splenic organogenesis marker expression, while molecular traits of TRC, MRC, and FDC were mostly absent in the PRC fraction (Supplementary Fig. 4f). Overall, these data support the notion that initiation of TRC/MRC/FDC subset specification and concomitant loss of stemness are the major LT $\beta \mathrm{R}$-dependent processes during white pulp formation.

To further decipher the differentiation trajectories of splenic FRC subsets and to determine the heterogeneity of splenic reticular cells at the single-cell level, we performed two different scRNA-seq analyses (Fig. 5a). The global scRNA-seq analysis of splenic fibroblastic stromal cells revealed eleven distinct cell clusters overlaid on a t-distributed stochastic neighboring embedding (tSNE) plot (Fig. 5b) with six clusters harboring cells that exhibited EYFP mRNA expression (Fig. 5c, d). Almost all cells in cluster 7 showed high Eng, Pdgfrb, and Ly6a expression, but were largely EYFP mRNA-negative (Fig. 5d). Confocal microscopy analysis revealed that Endoglin protein expression mainly highlighted red pulp fibroblasts and PRC of central arteries in the white pulp (Supplementary Fig. 5a). Expression of $C d 34$ was restricted to cells in clusters $8-11$, with mRNA of the extracellular matrix protein Lumican ( $\mathrm{Lum}$ ) highlighting clusters 10 and 11 and the mRNA of the mesothelial cell marker Mesothelin $(M s l n)$ being expressed only in clusters 8 and 9 (Fig. 5d). We found that Lumican ${ }^{+}$cells form a subcaspsular fibroblast layer (Supplementary Fig. 5b), while Mesothelin ${ }^{+}$cells form the outer layer of the splenic capsule (Supplementary Fig. 5c). Importantly, $\mathrm{EYFP}^{+}$cells were scarce in the subcapsular layer and completely absent in the mesothelial cell layer (Supplementary Fig. 5b, c). Utilizing the additional marker combinations derived from the global scRNA-seq analysis, we have elaborated a flow cytometry protocol that facilitates distinction of red pulp and white pulp fibroblastic stromal cells (Supplementary Fig. 5d). Overall, more than $80 \%$ of ICAM-1 ${ }^{+}$ $\mathrm{CD} 157^{+}$Endoglin $^{-}$white pulp fibroblasts expressed the EYFP marker (Supplementary Fig. 5d).

Next, we employed single-cell transcriptomic analysis on sorted $\mathrm{EYFP}^{+}$cells from Ccl19-iEYFP spleens. As expected, the scRNA-seq analysis revealed six distinct clusters overlaid on a tSNE plot with PRC, TRC, MRC, and FDC subsets (Fig. 5e, f) that could be identified through the gene signatures obtained by popRNA-seq (Supplementary Fig. 4). In accordance with the flow cytometry-based subset definition (Fig. 1), the two remaining clusters were assigned as triple negative cells (TNC1 and TNC2, respectively) (Fig. 5e). The TNC1 population showed enriched expression for canonical mural cell markers such as Pdgfrb, Cnn1, Cspg4 (also known as NG2), and Mcam (CD146) (Fig. 5g), while the TNC2 population showed highly upregulated genes that are involved in cell cycling such as Mki67, Mcm7, Cdk6, and Cks2 (Fig. 5h). Taken together, these transcriptome analyses unveil the heterogeneity of splenic white pulp FRC subsets and reveal the existence of additional cell populations that relate to the immuneinteracting reticular cells.

Differentiation trajectories of splenic FRC subsets. To corroborate FRC subset identity and to elucidate further details in the FRC differentiation trajectories, we incorporated EYFP ${ }^{+}$cells from adult Ccl19-iEYFP $L t b r^{\mathrm{fl} / \mathrm{fl}}$ spleens into the scRNA-seq analysis. The tSNE plot from Ltbr-deficient $\mathrm{EYFP}^{+}$cells confirmed the lack of TRC, MRC, and FDC differentiation and the accumulation of an extended PRC population, while both TNC populations were maintained (Supplementary Fig. 6a). Interestingly, an additional cell cluster appeared in Ltbr-deficient $\mathrm{EYFP}^{+}$ cells, designated as intermediate PRC (int-PRC) (Fig. 6a and Supplementary Fig. 6a). Int-PRC are characterized by the expression of PRC markers (e.g., Ly6a) and the lack of TRC/ MRC/FDC differentiation markers (e.g., Bst1, Enpp2, and Clu) (Supplementary Fig. 6b). Deconvolution of the FRC differentiation trajectories was achieved by employing the Monocle routine $^{33}$ that indicated PRC as progenitors for all differentiated white pulp reticular cell subsets (Fig. 6b). The Monocle analysis revealed two main differentiation trajectories with the LT $\beta R$ dependent branch leading toward TRC/MRC/FDC clusters and a LT $\beta$ R-independent branch with the TNC1 population, while int-PRC lined-up along both differentiation routes (Fig. 6b and Supplementary Fig. 6c). To further resolve the LT $\beta R$-dependent differentiation of TRC/MRC/FDC populations, we re-embedded the cells situated along the LT $\beta R$-dependent trajectory and re-employed the Monocle analysis. The Monocle trajectory of the LT $\beta R$-proficient cells demarcated the continued differentiation of PRC through the stage of int-PRC towards a first branching node (node 1) leading to a split into TRC vs. MRC/FDC populations (Fig. 6c). Along the $\mathrm{MRC} / \mathrm{FDC}$ route, a second node (node 2) mainly separated MRC from FDC subsets, albeit the absence of a distinct branch. In conjunction with the cell fatemapping experiments, these data indicate that the PRC fraction harbors postnatal mLTo cells that differentiate through an intermediate stage towards the TRC, FDC, and MRC in a LT $\beta R$-dependent fashion, while the maintenance of the periarterial PRC network is independent of this pathway.

To further decipher the relation of differentiated FRC subsets with LT $\beta R$-independent TNC subsets, we first screened the marker genes of PRC, int-PRC, and TNC1 populations based on the scRNA-seq analysis. Expression of mesenchymal stem cell markers such as Ly6a (Sca-1), Eng (CD105), Vcam1, and Pdgfra ${ }^{31,34}$ was highly enriched in PRC and int-PRC clusters, while the mural cell markers Mcam (CD146), Rgs5, Cspg4, and Cnn1 (Calponin) ${ }^{35}$ were mainly enriched in the TNC1 cluster 
a CCl19-iEYFP Ccl19-iEYFP $L t b r^{\text {fll/fl }}$

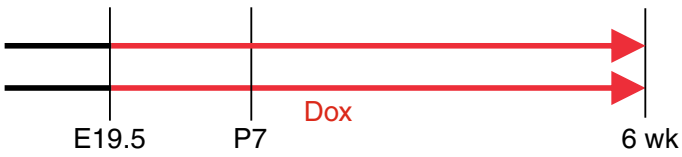

b Ccl19-iEYFP

d EYFP TER119 B220


C Ccl19-iEYFP $L t b r^{\mathrm{fl} / \mathrm{fl}}$

f EYFP TER119 B220
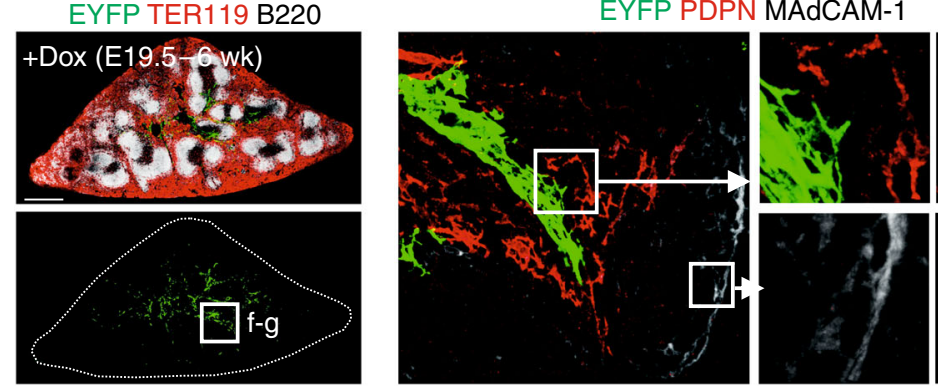

$\mathbf{h}$

Ccl19-iEYFP / +Dox E19.5-6 wk
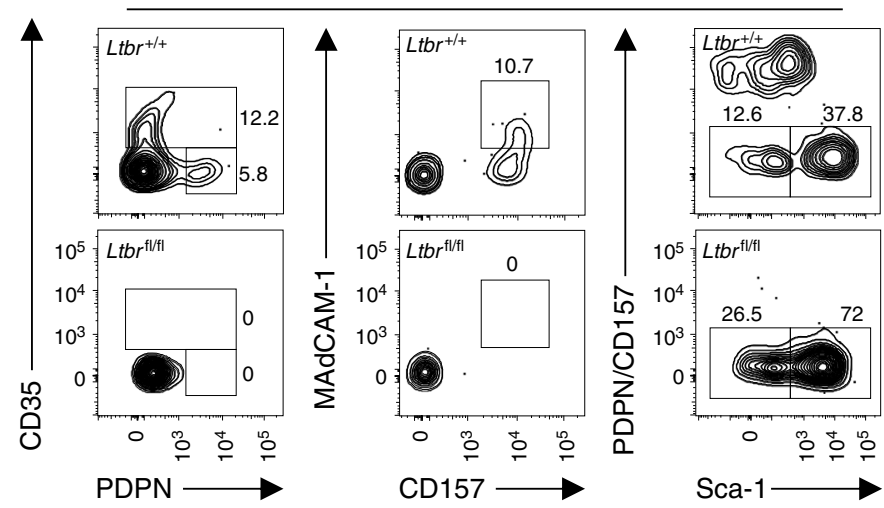

j



Ccl19-iEYFP / E19.5-6
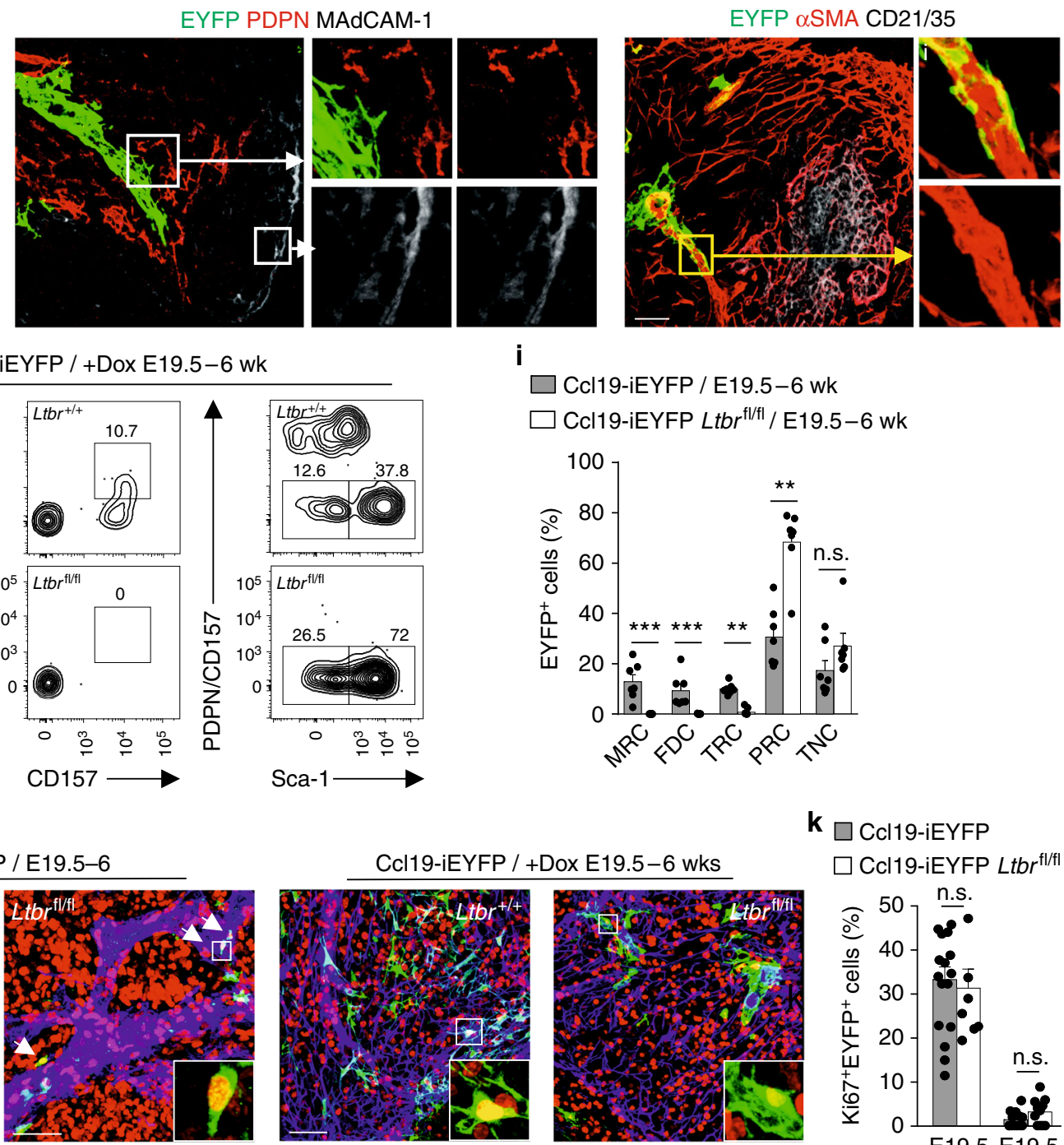

i

$\square$ Ccl19-iEYFP / E19.5-6 wk

$\square$ Ccl19-iEYFP $L t b r^{\mathrm{fl} / f \mathrm{l}} / \mathrm{E} 19.5-6 \mathrm{wk}$

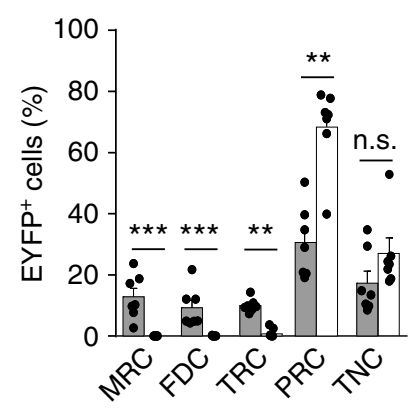

\section{$\mathbf{k} \square$ Ccl19-iEYFP}

$\square$ Ccl19-iEYFP $L t b r^{f l / f l}$
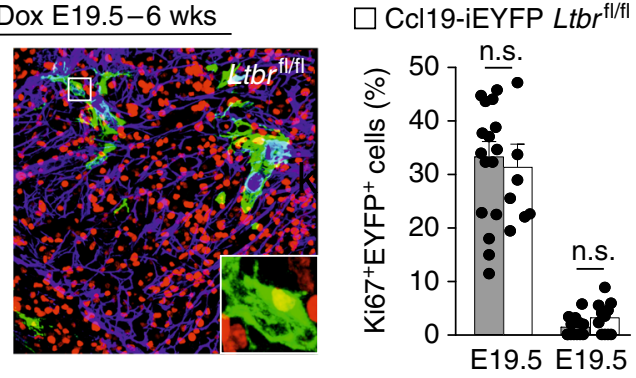

-6 wk

(Fig. 6d). Moreover, the expression of common perivascular cell markers Itgb1 (CD29) and Pdgfrb was upregulated at similar levels in the PRC, int-PRC, and TNC1 populations (Fig. 6d) suggesting that TNC1 represent mural cells. Flow cytometric analysis of $\mathrm{EYFP}^{+} \mathrm{CD} 157^{-}$reticular cells confirmed that TNC1 express CD29, but can be distinguished from PRC by the lack of Sca-1 expression and the lack of CD44 and reduced CD106 expression (Fig. 6e). Confocal microscopy revealed that CD29 ${ }^{+}$Calponin- $1^{+}$TNC1 form a distinct mural cell layer around white pulp arterioles (Fig. 6f). Gene-set enrichment analysis substantiated that this distinct mural cell subset contributes to blood vessel contractility and lacks the features 
Fig. 3 Cell fate mapping of embryonic lymphoid tissue organizer cells. a Schematic depiction of the experimental approach. Spleens have been harvested from E19.5 to 6 week fate mapped, b Ccl19-iEYFP, and c Ccl19-iEYFP Ltbr ${ }^{\mathrm{fl} / \mathrm{fl}}$ mice. Cross-sections were stained with the indicated antibodies and analyzed by confocal microscopy. Boxes indicate representative white pulp areas shown at higher magnification in (d-g) (scale bars $=500 \mu \mathrm{m})$. $\mathbf{d}$, e Confocal microscopy analysis of fate-mapped Ltbr-proficient EYFP+ white pulp reticular cell subsets highlighting PDPN+ TRC (d), MAdCAM-1+ MRC (d) and CD21/ $35^{+}$FDC (e), scale bars $=20 \mu \mathrm{m} / 5 \mu \mathrm{m}$. f, $\mathbf{g}$ Fate-mapped Ltbr-deficient EYFP+ cells by confocal microscopy using indicated marker combinations, scale bars $=20 / 5 \mu \mathrm{m}$. Microscopy data are representative for $n=7$ mice per group from three independent experiments. $\mathbf{h}$ Representative flow cytometric analysis of fate-mapped EYFP+ cells from Ccl19-iEYFP and Ccl19-iEYFP Ltbrl/fl spleens, gated on and analyzed for the indicated markers. Values indicate percentage of respective populations. i Percentage of EYFP + cells from spleens of indicated mouse strains, based on gating in (h) ( $n=7$ mice per group from three independent experiments, mean \pm SEM). j, k Assessment of cellular proliferation using Ki67 staining in spleens of Ccl19-iEYFP and Ccl19-iEYFP $L t b r^{\mathrm{fl} / \mathrm{fl}}$ mice at the indicated age with representative section in $(\mathbf{j})$ and quantification of the proportion of Ki67 expression in EYFP+ cells in $(\mathbf{k})(n=3-5$ mice per group from three independent experiments, mean \pm SEM). Statistical analyses were performed using a Mann-Whitney test. Source data are provided as Source Data file

a

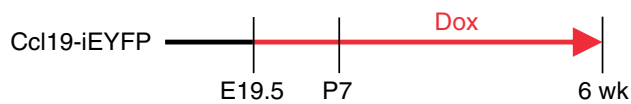

b
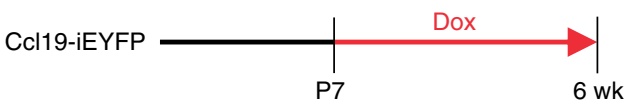

C
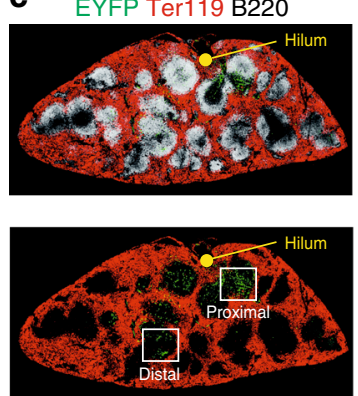

e

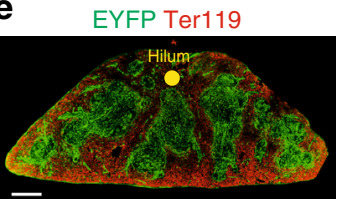

Concentric distance

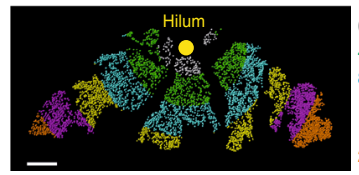

EYFP PDPN MAdCAM-1
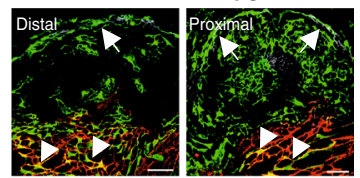

EYFP $\alpha$ SMA CD21/35

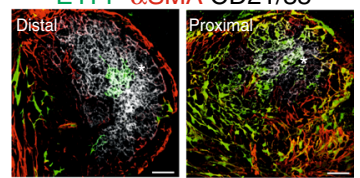

d
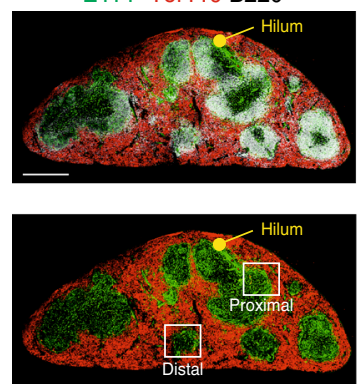

f
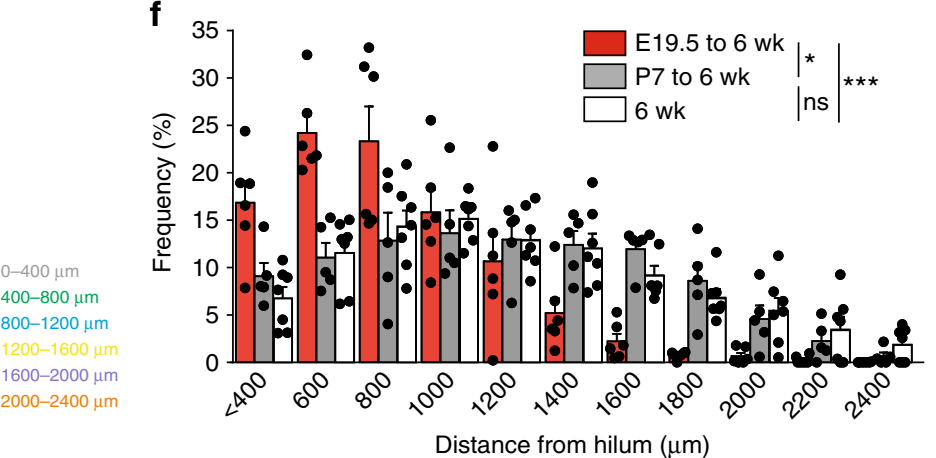

EYFP PDPN MAdCAM-1

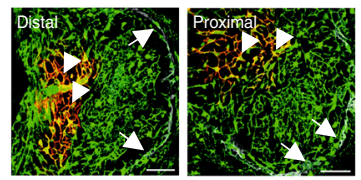

EYFP $\alpha$ SMA CD21/35

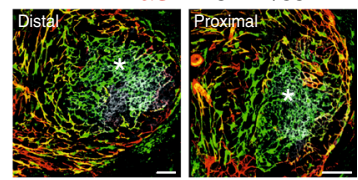

Fig. 4 Staggered commitment of splenic white pulp organizer cells along the developing arterial tree. $\mathbf{a}$, b Ccl19-iEYFP mice were treated with doxycycline from E19.5 (a, c) or P7 (b, d). Distribution and phenotype of EYFP+ cells in the white pulp of 6-week-old mice were determined by confocal microscopy using the indicated antibodies. Boxes show region of higher magnification in right images, arrows indicate EYFP+MAdCAM-1+ MRC $\mathbf{c}$, $\mathbf{d}$, arrowheads indicate EYFP+PDPN ${ }^{+}$TRC $(\mathbf{c}, \mathbf{d})$, asterisks indicate EYFP+CD21/35+ FDC $(\mathbf{c}, \mathbf{d})$ (scale bars $=500 / 20 \mu \mathrm{m}$ ). e Determination of EYFP ${ }^{+}$cell distances from the central artery at the hilum. Cross-sections from Ccl19-iEYFP spleens from 6-week-old mice were stained with antibody against TER119 and EYFP expression was pseudocolor-coded (lower image) according to concentric distance from the hilum (0-2400 $\mu \mathrm{m})$. Scale bar $=250 \mu \mathrm{m}$. f Frequency of EYFP + cells in white pulp areas of indicated concentric distances from the central artery $(n=5-7$ mice per group from two independent experiments, mean \pm SEM). Statistical analysis was performed using a Kolmogorov-Smirnov test. Source data are provided as Source Data file

and functions of the differentiated FRC subsets (Supplementary Fig. 6d). The TNC2 population was characterized by an enrichment of genes that are active during cell cycle and translation initiation (Supplementary Fig. 6d). Whether and how these proliferating $\mathrm{EYFP}^{+}$cells give rise to LT $\beta \mathrm{R}$-dependent or -independent fibroblastic stromal cell populations could not be resolved using the Monocle routine. In sum, our results indicate that adult PRC descend from embryonic periarterial mLTo cells and pass through an intermediate stage before developing into either mural cells or splitting up into the differentiation pathways of the main LT $\beta$ R-dependent reticular cell subsets (Fig. 6g).

\section{Discussion}

The current study has identified the embryonic origin and the differentiation trajectories of the adult reticular cell subsets that determine the structural organization of specialized immune environments in the splenic white pulp. Our findings reveal that stemness and multipotency are endowed to periarterial progenitor cells that contribute to the maintenance of the vascular structure and enable systemic immune surveillance in the splenic white pulp through subset specification of immune-interacting reticular cells.

Currently, competing views exist that promote divergent scenarios of reticular cell development in SLOs. Lymph node reticular 
a

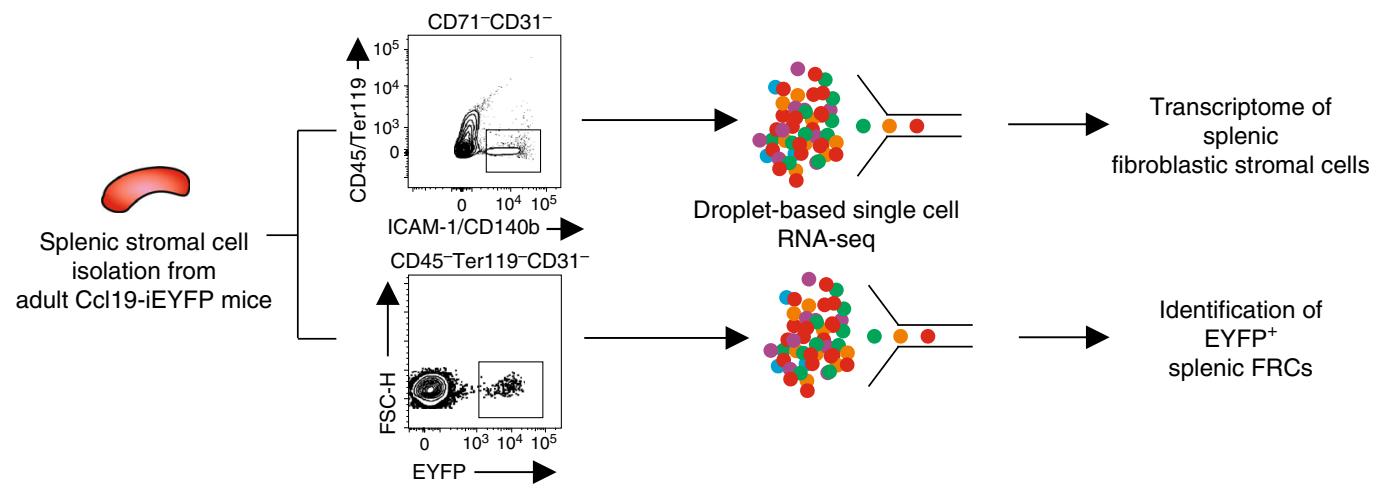

b
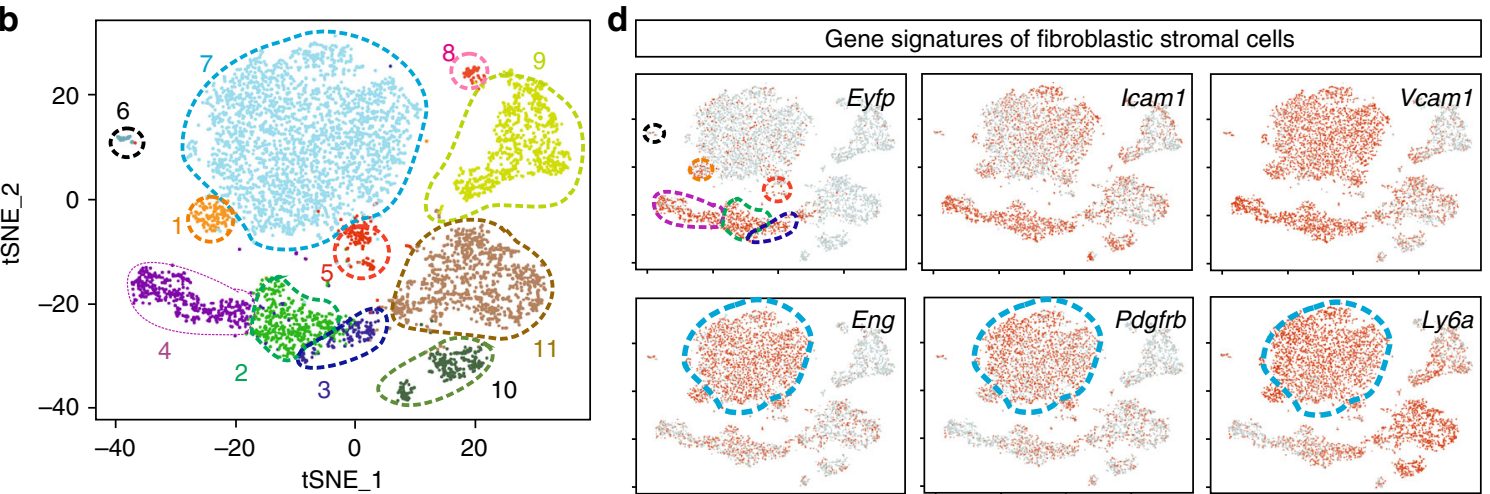

C
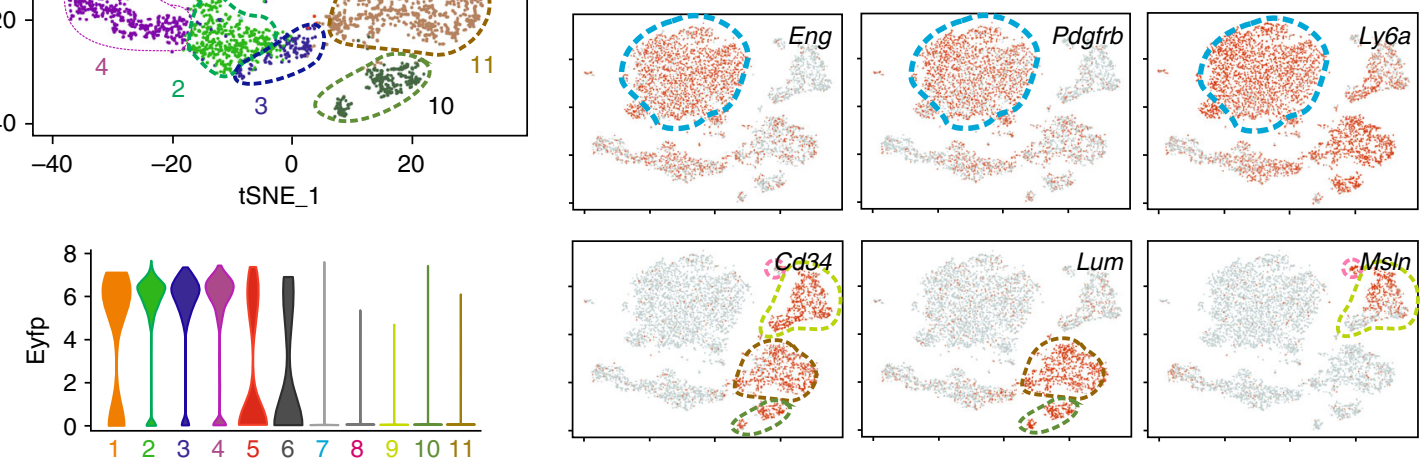

e
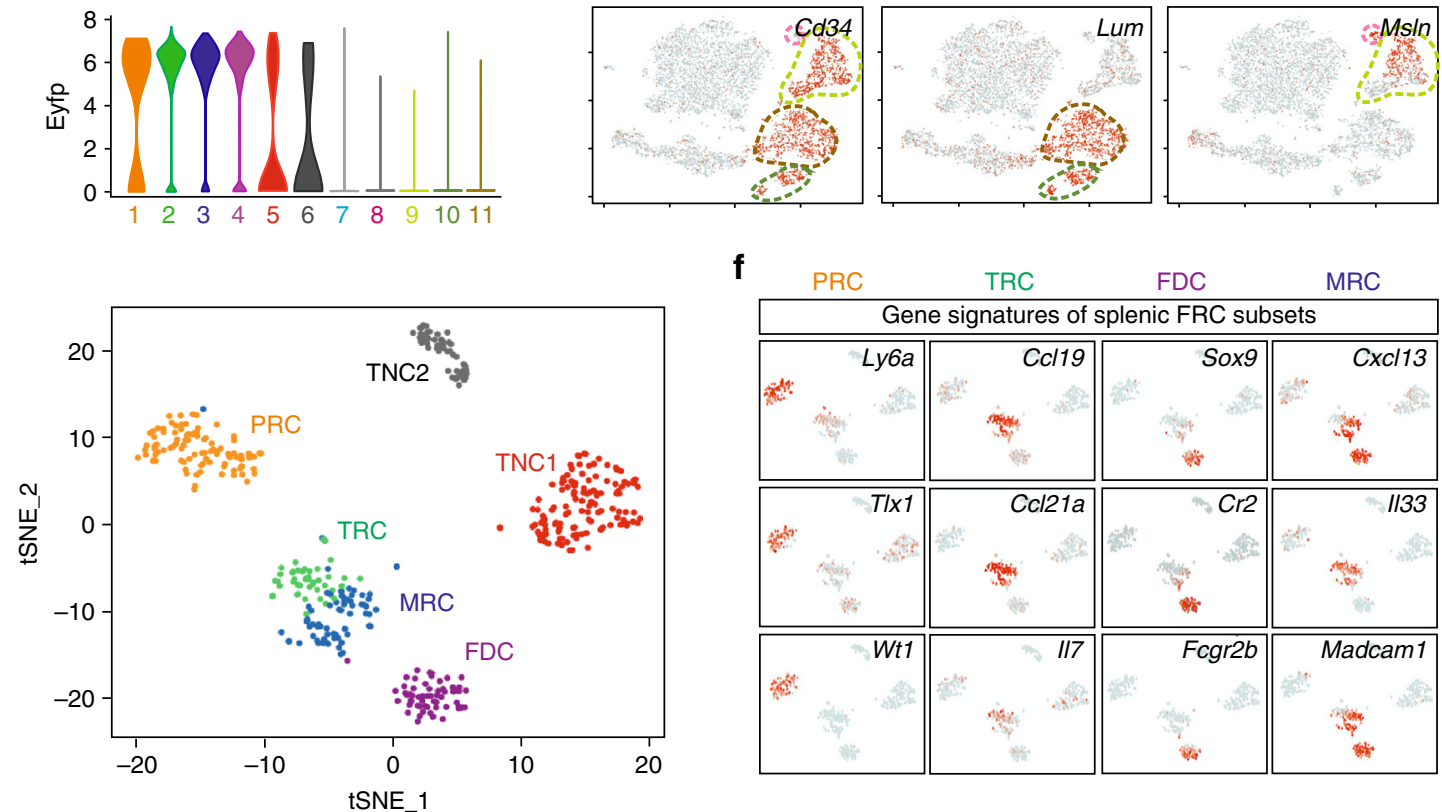

f

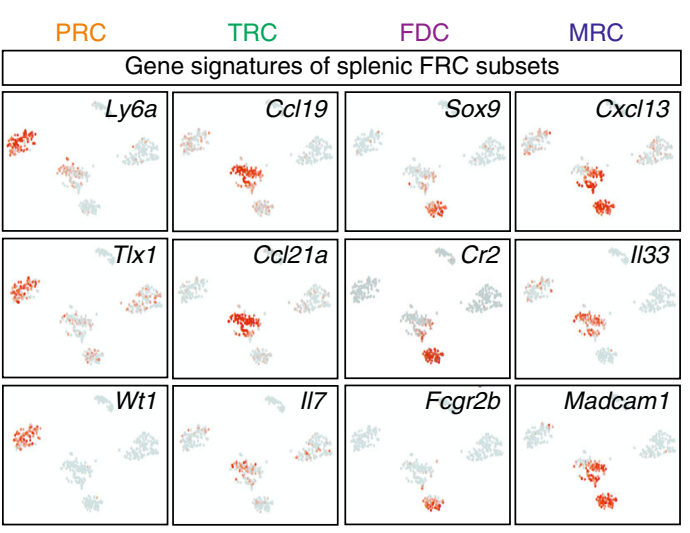

g
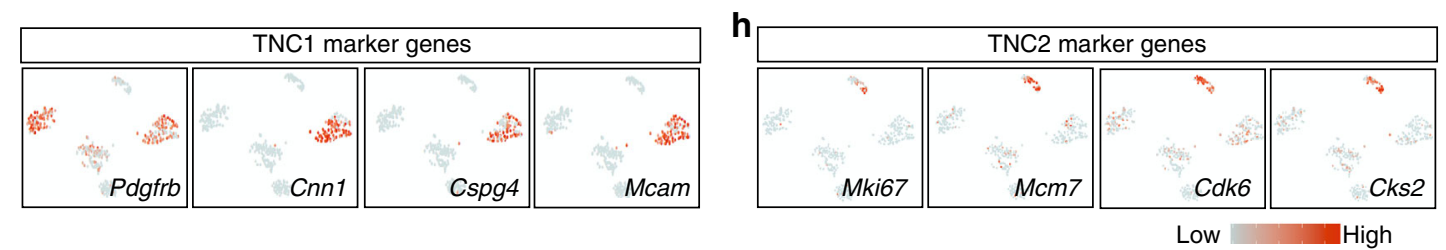

Fig. 5 Transcriptomic analysis of splenic white pulp reticular cells. a Schematic depiction of the droplet-based scRNA-seq workflow. $\mathbf{b}$ tSNE map of sorted splenic fibroblastic stromal cells from adult Ccl19-iEYFP mice. c Violin plots of Eyfp expression in different clusters based on the scRNA-seq analysis. d Gene signatures of distinct splenic reticular cell subsets. The density of the red color represents the expression level of the genes. e tSNE map of sorted EYFP ${ }^{+}$cells from adult Ccl19-iEYFP mice demarcated with the reticular cell populations. scRNA-seq data are pooled from two independent experiments. f-h Gene signatures of distinct splenic reticular cell subsets acquired from scRNA-seq analysis. The density of the red color represents the expression level of the genes 
a Merge of scRNA-seq
Ccl19-iEYFP and Ccl19-iEYFP $L t b r^{\text {fl/fl }}$

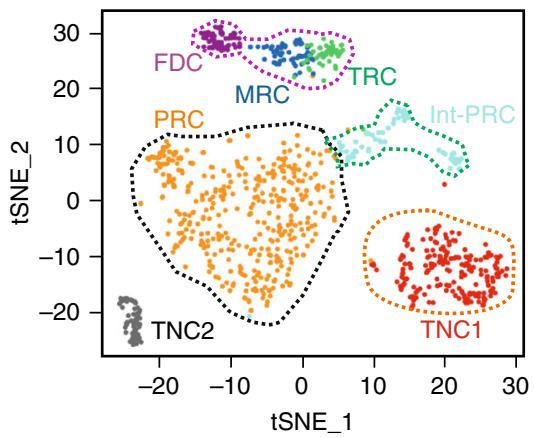

d

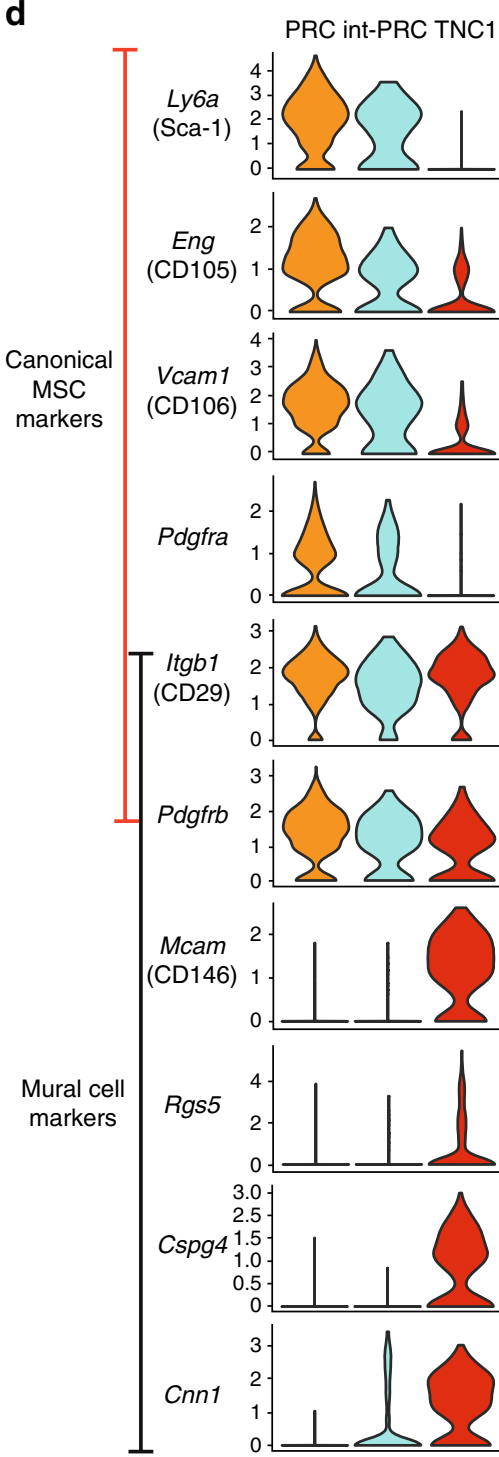

b

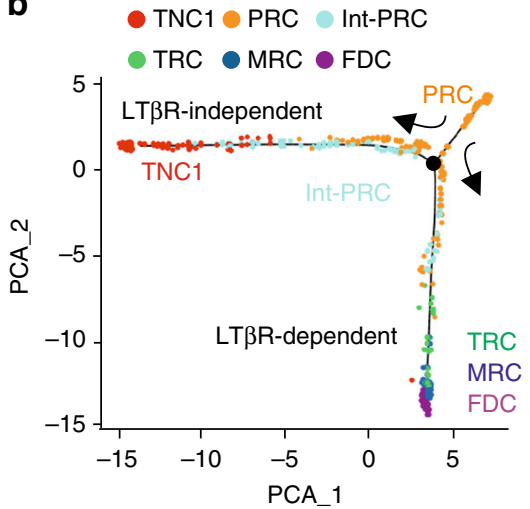

e CD45-Ter119- $^{-} \mathrm{EYFP}^{+}$
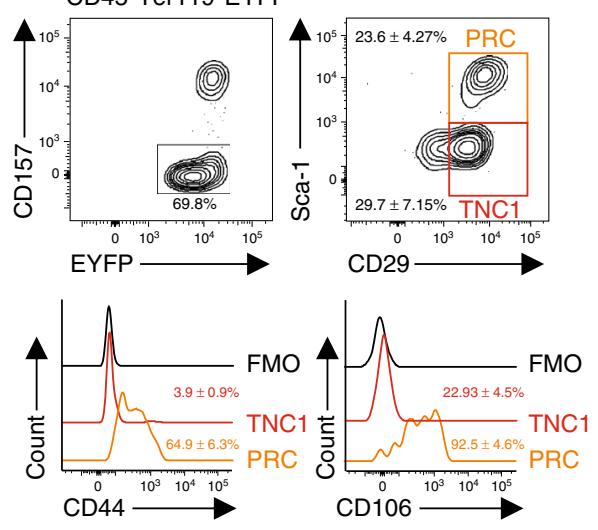
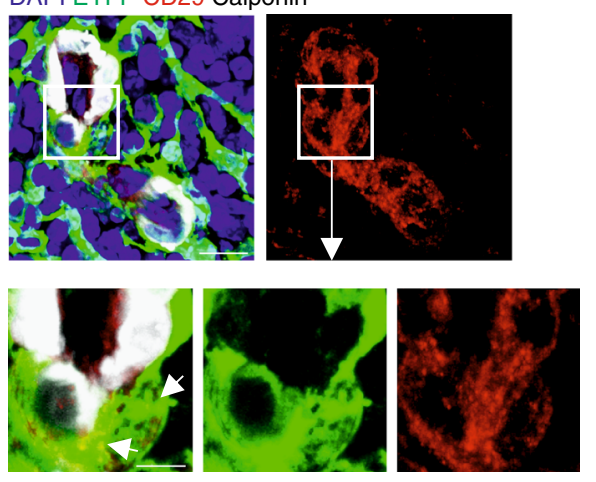

f DAPI EYFP CD29 Calponin

\section{g}

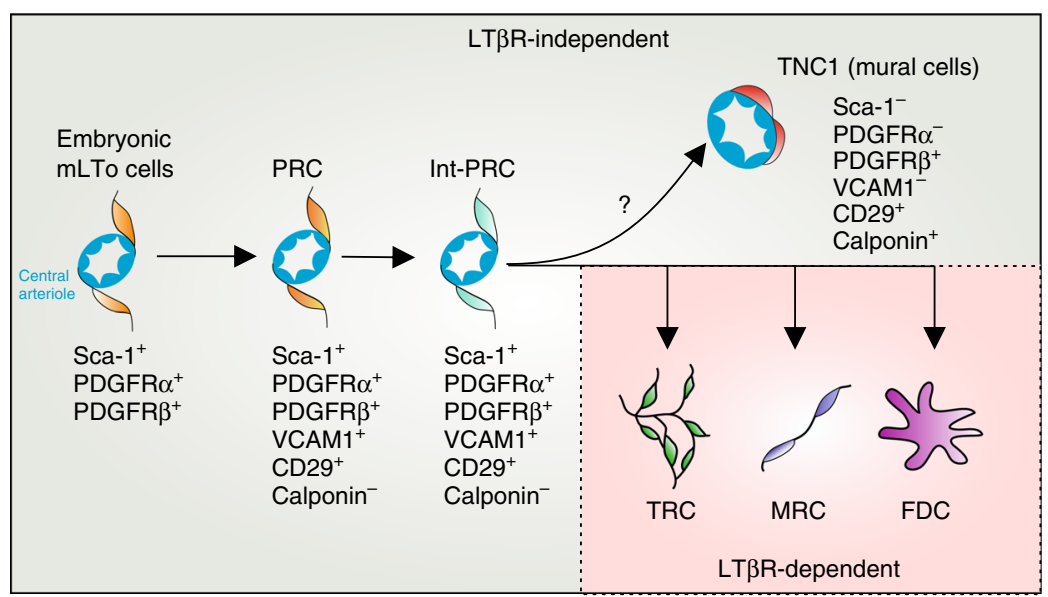

cells, for example, have been suggested to originate from preadipocyte progenitors ${ }^{36}$, which are triggered by retinoic acid from adjacent neurons to produce CXCL1 $3^{37}$. Based on the overlap in the expression of selected markers, MRC have been suggested to represent an intermediate mLTo cell ${ }^{38}$ and to give rise to $\mathrm{FDC}^{39}$. Assessment of SLO development in globally gene-deficient animals combined with heterotopic transfer of stromal cell populations has led to the conclusion that adventitial progenitors from SLOs can give rise to one $e^{40}$ or several reticular cell subsets ${ }^{41}$. Moreover, an endothelial cell-MRC hybrid cell has been proposed to function as key organizer cell during postnatal spleen regeneration ${ }^{42}$. These profound conceptual discrepancies could be resolved here through the generation of a cell-fate mapping system that targets the committed progenitors of the main reticular cell populations. Importantly, the Ccl19-iEYFP model excludes tracing of irrelevant mesenchymal cell lineages such as red pulp fibroblasts ${ }^{18}$ or myocardial smooth muscle cells ${ }^{22}$. Fate mapping of the relevant cell population has unveiled a discrete embryonic periarterial 
Fig. 6 Differentiation trajectories of reticular cell subsets in the splenic white pulp. a Merged tSNE cell map of sorted splenic EYFP+ cells from adult Ccl19iEYFP and Ccl19-iEYFP Ltbr ${ }^{f l / f l}$ spleen highlight the distinct reticular subsets. b Differentiation trajectory analysis of reticular cell populations constructed by the Monocle routine. Single cells are ordered along the trajectory tree according to the pseudo-time development. c Re-embedding of cell clusters along the LT $\beta R$-dependent trajectory re-analyzed with Monocle. Nodes indicate the bifurcating branching points of distinct cell populations along the differentiation trajectories. d Violin plots of canonical mesenchymal stem cell and mural cells markers expressed by PRC, int-PRC, and TNC1. e Representative flow cytometric analysis of EYFP+ cells from adult Ccl19-iEYFP spleen, gated on and analyzed for the indicated markers. Values indicate percentage of respective populations. ( $n=6$ mice per group from two independent experiments, mean \pm SEM). $\mathbf{f}$ Spleen cross-sections from adult Ccl19-iEYFP mice analyzed by confocal microscopy after staining with the indicated antibodies (scale bar $=10 \mu \mathrm{m}$ ). Boxed areas in (f) indicate the magnified region below (scale bar $=5 \mu \mathrm{m}$ ). $\mathbf{g}$ Schematic of the LT $\beta R$-independent and -dependent differentiation trajectory proposed for embryonic mLTo cells and postnatal PRC, and differentiated reticular cell populations. Source data are provided as Source Data file

progenitor niche for all reticular cell subsets. Stemness and multipotency of the periarterial progenitors were maintained through adulthood providing a source for both FRC subsets and mural cells. The distinct, LT $\beta$ R-dependent differentiation trajectories of TRC, MRC, and FDC suggest that these subsets integrate different sets of signals to generate suitable microenvironments of the diverse immune cell populations. Whether MRC represent a progenitor state 39 or a separate differentiation path could not be finally resolved in our analysis. Nevertheless, the Ccl19-iEYFP model will permit further dissection of reticular cell functions and differentiation pathways through cell-type specific and timed ablation of key molecules at different developmental stages and during ongoing immune responses.

Multipotent progenitor cells within the perivascular niche are usually referred to as mesenchymal stem cells ${ }^{43}$ because these cells exhibit osteogenic, chondrogenic, and adipogenic potential $^{31,44}$ and contribute to pathologic fibrosis in multiple organs ${ }^{45}$. Perivascular stem cells are characterized by the expression of PDGFR $\alpha$, PDGFR $\beta$, and Sca-1 ${ }^{31,44}$, markers that are also expressed by PRC as shown in this study. The finding that the expression of the pericyte markers CD146 and NG2, encoded by Mcam and Cspg4, respectively, is associated with multipotent differentiation potential ${ }^{44}$ has led to the conclusion that pericytes can function as mesenchymal stem cells. Recent studies, however, have challenged this view because even though pericytes exhibit tri-lineage differentiation in vitro, these properties could not be confirmed using in vivo cell-fate mapping ${ }^{46}$. Our finding that mural cells designated as TNC1 express the classical pericyte markers NG2, CD146, and RSG5 ${ }^{35}$, while the PRC and int-PRC were negative for these markers, support the conclusion that splenic reticular cells do not originate from pericytes. Clearly, splenic periarterial PRC possess multipotent stem cell functions and can be programmed to differentiate either into cells that promote vascular wall stability or give rise to different reticular cell subsets in a process that depends on signals provided in the respective microenvironment.

The evolution of SLOs provides important insight into how reticular cell subset specialization has followed developmental programs of lymphocytes. While specific antigen receptor-bearing cells appeared some 500 million years ago in a common vertebrate ancestor ${ }^{47}$, lymph nodes with their fully developed set of functional microenvironments are a rather late amendment to the immune system which emerged $\sim 100$ million years ago in mammals ${ }^{48}$. In particular, the evolution of the spleen from amphibians to birds, starting $\sim 300$ million years ago, illustrates the adaptation of the white pulp infrastructure to the developing adaptive immune system ${ }^{49}$. Amphibians such as Xenopus laevis possess a distinguishable white pulp with lymphocytes forming concentric layers around the central arterioles and the white pulp in reptiles is underpinned by reticular cells immediately adjacent to the arteriole ${ }^{49}$. In subsequent evolutionary steps in birds, the splenic white pulp developed bursa-dependent germinal centers $^{50}$ that are characterized by the presence of FDC ${ }^{51}$. Hence, gain of more complex functions of lymphocytes, such as affinity maturation in germinal centers, was accompanied by the coevolution of the suitable microenvironments that are built by specific reticular cell niches. Such cellular interactions require hard-wired signaling circuits that permit on-demand formation of the suitable stromal cell infrastructure.

The molecular dissection of the reticular cell subsets indicates that a basic two-signal program drives TRC/MRC/FDC subset specification and thereby shapes the distinct microenvironments in the white pulp. It appears that mLTo cell activation via the LT $\beta R$ signaling functions as essential "signal 1 " that needs to be delivered to initiate and to ensure specificity of the process. The existence of a mandatory first signal resembles the two-signal principle underlying lymphocyte activation, which requires the encounter with the cognate antigen as "signal 1"52. Accordingly, "signal 2" components need to be present to secure, for example, T-cell zone- vs. B-cell zone-specific reticular cell differentiation pathways. It is conceivable that second signals guiding reticular cell subset specialization reflect extrinsic imprints delivered by lymphocyte subsets in their immune microenvironments. Such context-specific integration of secondary differentiation signals by reticular cells may involve other members of the TNF receptor superfamily, which serve as secondary signals during $\mathrm{T}$ - and Bcell differentiation ${ }^{53}$. Interestingly, both naive $\mathrm{T}$ and $\mathrm{B}$ cells, i.e., the progenitors of effector lymphocytes, are maintained in the absence of specific antigenic stimulation by a process known as homeostatic proliferation 54,55 . Likewise, postnatal reticular progenitors were maintained and proliferated at a low frequency in the absence of LT $\beta R$ signaling suggesting that yet undefined "signal 0" processes are sufficient to nourish the progenitor niches. Hence, while homeostatic signals are required to maintain the pool of reticular cell progenitor cells, the differentiation trajectories of reticular cell subsets reveal the fundamental twosignal principle that governs the structural and functional integrity of lymphoid organs.

The primary function of LT $\beta$ R signaling in reticular cell differentiation has been demonstrated in mice that lack $L t b r$ expression in Ccl19-expressing cells ${ }^{13}$, where reticular cell subsets fail to differentiate and remain arrested in the myofibroblastic progenitor stage. Second signals required for subset specification can be delivered by other molecules of the TNF receptor superfamily. For example, ablation of TNF in B cells prevents FDC differentiation $^{56}$, while the lack of CD30 contributes to abnormal $\mathrm{B}$ - and T-cell zone segregation that is associated with loss of podoplanin expression ${ }^{15}$. The massive extension of the complex receptor-ligand system of the TNF receptor superfamily that has accompanied the development of the adaptive immune system in jawed vertebrates ${ }^{57}$ further supports the notion of an evolution of discrete signaling hierarchies in reticular cell differentiation. The advent of highly specialized immune environments in lymph nodes of mammals ${ }^{48}$ has probably made LT $\beta R$ the master regulator that controls the initiation of reticular cell subset specification. In conclusion, the concept that discrete signaling levels in perivascular niches determine the nature of reticular cell networks provides a theoretical framework for further exploration of 
innate and adaptive immune processes in secondary and tertiary lymphoid tissues.

\begin{abstract}
Methods
Mice. All mouse strains were on a C57BL/6NCrl genetic background and maintained in individually ventilated cages under specific pathogen free conditions. The BAC-transgenic C57BL/6N-Tg(Ccl19-tTA) ${ }^{688 \mathrm{BIAT}}$ (Ccl19-tTA) mouse was generated using homologous recombination-mediated transgenesis ${ }^{58}$ using a tetracycline transactivator-based single-cassette system for spatiotemporal gene regulation ${ }^{59}$. The $\mathrm{C} 57 \mathrm{BL} / 6 \mathrm{~N}-\mathrm{Tg}(\mathrm{Ccl} 19-\mathrm{Cre}) 489 \mathrm{Biat}(\mathrm{Ccl} 19-\mathrm{Cre})^{13}$ and the $\mathrm{Ltbr}^{\mathrm{tm} 1.1 \mathrm{Thhe}}\left(L T \beta R^{f l / f l}\right){ }^{60}$ strains were described previously. B6.129 $\times 1-G t(R O S A)$ $26 \operatorname{Sor}^{\text {tml } 1(E Y F P) \operatorname{Cos} / J ~(R 26 R-E Y F P) ~ a n d ~ B 6.129 ~ \times ~ 1-G t(R O S A) 26 S o r ~}{ }^{\text {tm1(CAG-Brain- }}$ bow2.1)Cle (Brainbow2.1) $)^{32}$ mice were purchased from The Jackson Laboratories and the LC-1 strain $^{26}$ was kindly provided by Dr. Fendler, Max Delbrück Center of Molecular Medicine, Berlin, Germany. Fate-mapping experiments were performed via treating the pregnant dam with $1 \mathrm{mg}$ doxycycline (Sigma Aldrich) intraperitoneally and maintenance of doxycycline in the drinking water $(1 \mathrm{mg} / \mathrm{ml})$. Experiments were performed in accordance with Swiss federal and cantonal guidelines (Tierschutzgesetz) under the permission numbers SG13/15, SG16/07 and SG05/17 granted by the Veterinary Office of the Canton of St. Gallen.
\end{abstract}

Preparation of splenic stromal cells. Spleens were harvested and perfused with RPMI 1640 medium containing 2\% FCS, $20 \mathrm{mM}$ Hepes (all from Lonza), $0.2 \mathrm{mg} /$ $\mathrm{ml}$ Collagenase P (Sigma Aldrich), 0.8 U/ml Dispase I (Sigma Aldrich) and $100 \mu \mathrm{g} /$ $\mathrm{ml}$ DNaseI (Applichem) with $22 \mathrm{G}$ syringe. Samples were torn into smaller pieces and incubated at $37^{\circ} \mathrm{C}$ for $30 \mathrm{~min}$, with resuspension and collection of supernatant every $15 \mathrm{~min}$ to PBS containing $1 \%$ FCS and $10 \mathrm{mM}$ EDTA (MACS buffer). To enrich fibroblastic stromal cells, hematopoietic and erythrocytes were depleted by incubating the cell suspension with MACS anti-CD45 and anti-TER119 microbeads (Miltenyi Biotec) and passing them through a MACS LS column (Miltenyi Biotec). Unbound single-cell suspensions were used for further flow cytometric analysis.

Flow cytometry. Single-cell suspensions were incubated for 20 min at $4{ }^{\circ} \mathrm{C}$ in PBS containing $1 \%$ FCS and $10 \mathrm{mM}$ EDTA with the following antibodies: CD3 (clone:145-2C11, BioLegend at 1:200, Cat: 100305), CD19 (clone: eBio1D3, Thermo Fischer Scientific at 1:200, Cat: 25-0193-82), CD127 (clone:SB/199, BioLegend at 1:100, Cat: 121121), ROR $\gamma t$ (clone:B2D, Thermo Fischer Scientific at 1:100, Cat: 46-6981-82), CD45 (clone:30-F11, BioLegend at 1:200, Cat: 103113), CD71 (clone: RI7217 at 1:200, Cat: 113811), CD157 (clone:BP-3, BioLegend at 1:200, Cat:140207), Ter119 (clone:TER-119, BioLegend at 1:200, Cat: 747740), CD31 (clone:MEC13.3, BioLegend at 1:200, Cat: 102419), PDPN (clone:8.1.1, BioLegend at 1:200, Cat: 127407 or 127417), CD35 (clone:8C12, BD Bioscience at 1:200, Cat: 553816), MAdCAM-1 (clone:MECA-367, BioLegened at 1:100, Cat: 120705), CD140a (clone:APA5, BioLegend at 1:100, Cat: 135909), Sca-1 (clone:D7, BioLegend at 1:100, Cat: 122523), CD29 (clone: HMbeta1-1, BioLegend at 1:200, Cat: 102225), CD106 (Clone: 429, BD Bioscience at 1:200, Cat: 740865), Endoglin (Clone: MJ7/18, BD Bioscience at 1:200, Cat: 564746), CD44 (Clone: IM7, BioLegend at 1:200, Cat: 103011). Streptavidin-conjugated PE-Cy7 (BioLegend at 1:1000, Cat: 405206) have been used to detect biotinylated antibodies. Cells were acquired with a FACS Canto II or BD LSR Fortessa (BD Biosciences) and analyzed using FlowJo software v7 or v10 (Tree Star Inc.).

Histology. Spleen samples were fixed overnight with $4 \%$ paraformaldehyde (Merck) in PBS under agitation at $4{ }^{\circ} \mathrm{C}$. Fixed samples were washed with PBS containing 1\% TritonX-100 (Sigma) and 2\% fetal calf serum (Sigma) overnight at 4 ${ }^{\circ} \mathrm{C}$. Samples were embedded in $4 \%$ low-meting agarose gel (VWR International) and were sectioned into $40 \mu \mathrm{m}$ using vibratome (Leica VT-1200). Sections were blocked in PBS containing 1\% TritonX-100 (Sigma), 10\% fetal calf serum (Sigma) and $1 \mathrm{mg} / \mathrm{ml}$ anti-Fcy receptor (BD Biosciences) at $4{ }^{\circ} \mathrm{C}$ for $2 \mathrm{~h}$ and further incubated overnight with the following antibodies: anti-CD157 (BioLegend at 1:200, Cat:140207), anti-PDPN (BioLegend at 1:500, Cat: 127401), anti-CD4 (BioLegend at 1:500, Cat: 100403), anti-B220 (BioLegend at 1:500, Cat: 103229), anti-aSMA (Sigma at 1:500, Cat: C6198), anti-EYFP (Takara at 1:500, Cat:632592), anti-CCL21 (R\&D Systems at 1:100, Cat:AF457), anti-CXCL13 (R\&D Systems at 1:100, Cat: AF470), anti-MAdCAM-1 (clone:MECA-367, BioLegened at 1:100, Cat: 120705), anti-CD21/35 (BD Bioscience at 1:100), anti-CD35 (clone:8C12, BD Bioscience at 1:100, Cat: 553816), anti-TER119 (BioLegend at 1:500, Cat: 116203), anti-CD29 (clone: HMbeta1-1, BioLegend at 1:100, Cat: 102203), anti-Calponin-1 (clone: EP798Y, abcam at 1:100, Cat:ab46794), anti-Lumican (R\&D systems at 1:200, Cat:AF2745), ant-Methoselin (Invitrogen at 1:100, Cat:PA5-79698), Endoglin (BD Bioscience at 1:100, Cat: 550546) and anti-tdTomato (Takara at 1:500, Cat:632496). Unconjugated antibodies were detected with the following secondary antibodies: Alexa488-conjugated anti-rabbit-IgG (at 1:500, Cat: 711-005-152), Dylight549conjugated anti-rat-IgG (at 1:500, Cat: 712-165-153), Dylight549-conjugated antisyrian hamster-IgG (at 1:500, Cat: 307-506-003), Cy3-conjugated Streptavidin (at 1:500, Cat: 016-160-084), Alexa647-conjugated Streptavidin (at 1:1000, Cat: 016-600-084), Alexa647-conjugated anti-goat-IgG (at 1:500, Cat: 705-606-147) (all purchased from Jackson Immunotools). For Sca-1 and CD29 staining, splenic tissue was fixed for $2 \mathrm{~h}$ with $4 \%$ paraformaldehyde in PBS under agitation at $4^{\circ} \mathrm{C}$ Samples were incubated in $30 \%$ sucrose overnight at $4{ }^{\circ} \mathrm{C}$ and were embedded in OCT (VWR International) and frozen on dry ice. OCT-embedded samples were sectioned into 6-8 $\mu \mathrm{m}$ thickness using a cryostat (MICROM HM $500 \mathrm{OM}$ ). Sections were blocked with PBS containing $1 \%$ TritonX-100 (Sigma), $10 \%$ fetal calf serum (Sigma) and $1 \mathrm{mg} / \mathrm{ml}$ anti-Fc $\gamma$ receptor (BD Biosciences) at $4{ }^{\circ} \mathrm{C}$ for $2 \mathrm{~h}$ and further incubated overnight with biotin-conjugated anti-Sca-1 (BioLegend at 1:100, Cat: 108103) or Biotin-conjugated anti-CD29 (BioLegend at 1:100, Cat: 102203) followed by tyramide signal amplification kit (Life Technologies). For whole mount tissue acquisition, spleens were fixed overnight with $4 \%$ paraformaldehyde in PBS at $4{ }^{\circ} \mathrm{C}$, washed twice with PBS containing $1 \%$ TritonX-100 (Sigma), $10 \%$ fetal calf serum (Sigma) and $1 \mathrm{mg} / \mathrm{ml}$ anti-Fc $\gamma$ receptor (BD Biosciences) for $2 \mathrm{~h}$ and incubated with anti- $\alpha \mathrm{SMA}-\mathrm{Cy} 3$ antibodies overnight. After three more washing steps splenic tissues were cleared with FocusClear (CelExplorer) and directly imaged. Microscopy analysis was performed using a confocal microscope (Zeiss LSM-710) and images were processed with ZEN 2010 software (Carl Zeiss, Inc.) and Imaris (Bitplane).

Quantification of Ki67 ${ }^{+}$cells by histology. Spleens from Ccl19-iEYFP mice were sectioned at embryonic day E19.5, P0, P7, 2 week and 6 week. Samples were stained for the following markers: DAPI (Thermo Fischer Scientific), EYFP, $\alpha$-SMA and Ki-67 (Clone; SolA15, Thermo Fischer Scientific at 1:500, Cat: 13-5698-82). One to five white pulp data sets were generated per mouse. 3D Z-stack images of the sections were acquired by high-resolution confocal microscopy. $\mathrm{EYFP}^{+}$reticular cells were $3 \mathrm{D}$ reconstructed in Imaris (Bitplane) and the 3D isosurfaces were masked to the DAPI and Ki-67 channels separately in order to generate DAPI ${ }^{+}$ and Ki- $67^{+}$nuclei belonging to reticular cells specifically. Total numbers of $\mathrm{DAPI}^{+}$ reticular cells and $\mathrm{Ki}-67^{+}$reticular cells per white pulp were calculated using the Spots object generation. Any false positives were manually removed from analysis. Likewise, false negatives were minimized by manually adding spots to the reticular cells that were not detected by the Spots algorithm in Imaris. The results of the analysis were displayed as percentage of activated Ki- $67^{+}$cells from the total number of reticular cells in the splenic white pulp.

Splenic reticular cell sorting, library preparation and RNA-seq analysis. EYFP + cells from Ccl19-iEYFP fetal spleens (mean yield $=1150$ cells, $n=5$ ), adult Ccl19-iEYFP (mean yield $=4100$ cells, $n=7$ ) and Ccl19-iEYFP Ltbrfl/fl (mean yield $=3500$ cells, $n=9$ ) spleens were sorted with a Bio-Rad S3 cell sorter and were collected in $200 \mu \mathrm{l}$ Eppendorf tubes containing $100 \mu \mathrm{l}$ of RNAlater reagent (Qiagen) to preserve the RNA after sorting. Likewise, reticular cell populations were sorted based on the defined markers sets, i.e., $\mathrm{PDPN}^{+}$TRC (mean yield $=670$ cells, $n=4$ ), CD $35^{+}$FDC (mean yield $=950$ cells, $n=3$ ), MAdCAM $-1^{+}$MRC (mean yield $=1010$ cells, $n=2$ ), Sca- ${ }^{+}$PRC (mean yield $=1525$ cells, $n=3$ ). RNA extraction was performed using Quick-RNA Mini-Prep (Zymo Research). Reverse transcription and cDNA library generation was done using the Ovation SoLo RNA-Seq System (NuGEN) as described by the manufacturer. cDNA libraries were quantified by KAPA library Quantification kit (KAPA Biosystems) and Agilent Tape station in the Functional Genomics Center Zurich, Zurich, Switzerland. Sequencing of the cDNA libraries was performed on a Illumina HiSeq 2500 by the Functional Genomics Center Zurich.

RNA-seq data processing and analysis. Single-end reads (126 nt) were trimmed with Trimmomatic $\mathrm{v} 0.33^{61}$ and flexbar $\mathrm{v} 2.5^{62}$ to remove adapters and low-quality bases. The trimmed reads were aligned to the mouse genome (GRCm38) with STAR v2.5.2a $\mathrm{a}^{63}$ and deduplicated with NuDup v2.2. Reads that start at the same genomic coordinate and have the same strand orientation and $8 \mathrm{nt}$ molecular tag sequence were considered duplicates. The deduplicated BAM files were converted back to FASTQ format using bedtools bamtofastq v2.17.0 and duplicate read entries were removed. A transcriptome index was generated from the combined cDNA and ncRNA sequence files (Ensembl GRCm38.82) and Salmon v0.7.1 ${ }^{64}$ was used to estimate transcript abundances, which were read into R (v3.4.0) and summarized on the gene level with tximport (v1.4.0), together with average transcript length offsets ${ }^{65}$. Six samples with low-mapping rate from Salmon $(<30 \%)$ and/or poor strand specificity were excluded from further analysis. For differential expression analysis, only genes with estimated CPM above 0.5 in at least 2 samples were retained and a generalized linear model with analysis date (batch) and cell type as predictors was fit to each gene using edgeR (v3.18.1 $)^{66}$. Two groups of contrasts were tested using edgeR's LRT framework: the first group contains the three pairwise comparisons between the embryonic $\mathrm{EYFP}^{+}$cells, Ltbr-proficient and $L t b r$-deficient $\mathrm{EYFP}^{+}$adult cells, and the second group contains the ten pairwise comparisons between embryonic $\mathrm{EYFP}^{+}$cells as well as the PRC, MRC, TRC, and FDC subsets. $p$ Values were adjusted for multiple comparisons using the Benjamini-Hochberg method within each contrast and genes with FDR-adjusted $p$ value $<0.05$ were considered significantly differentially expressed. For each group of contrasts, MDS plots were generated from the $\log C P M$ values (calculated with the cpm function of edgeR with a prior count of 2) of the genes that were differentially expressed for at least one of the included contrasts (adjusted $p$ value $<$ 0.05 ), using the plotMDS function of the limma package (v3.32.2). The camera 
function from the limma package ${ }^{67}$ was used to perform gene set analysis for the first group of contrasts, considering the $\mathrm{C} 2, \mathrm{C} 5$, and $\mathrm{C} 7$ gene-set collections from mSigDB (v5.2) downloaded from http://bioinf.wehi.edu.au/software/MSigDB/. The $p$ values were calculated using the competitive gene set test accounting for intergene correlation within the camera function. Individual gene expression heatmaps display $\log$ CPM values, incorporating TMM normalization factors, followed by gene-wise $Z$-score standardization and, where indicated, averaging over all samples within a group.

Droplet-based single-cell RNA-seq analysis. Total splenic fibroblastic stromal cells were sorted with a Bio-Rad S3 cell sorter based on the fibroblast markers ICAM-1, PDGFR $\beta$, CD157, and PDPN from Ccl19-iEYFP adult spleens and single cell suspensions were run on the $10 \times$ Chromium analyzer $\left(10 \times\right.$ Genomics ${ }^{68}$. Likewise, $\mathrm{EYFP}^{+}$cells from Ccl19-iEYFP and Ccl19-iEYFP $L t b r$ fllfl adult spleens were sorted with a Bio-Rad S3 cell sorter and were run on the 10× Chromium analyzer $(10 \times \text { Genomics })^{68}$. The cDNA library generation was performed by the functional genomic center Zurich following the established commercial protocol for Chromium Single Cell 3' Reagent Kit (v2 Chemistry). Libraries were run via Hiseq2500 rapid run for Illumina sequencing. Samples containing total splenic fibroblastic stromal cells and only $\mathrm{EYFP}^{+}$cells were analyzed separately. Preprocessing and gene-expression estimation was performed using CellRanger (v2.1.1) ${ }^{69}$, generating UMI counts for 8364 cells and 26,596 genes with a count above zero in at least one cell for samples containing total splenic fibroblastic stromal cells and 1852 cells and 24,796 genes from EYFP ${ }^{+}$samples. The reference files used to build the index were obtained from the Ensembl GRCm38.90 release. Initial quality control was done with the scater R/Bioconductor package $(\mathrm{v} 1.6 .3)^{70}$ running in $\mathrm{R}$ v3.4.2. In order to remove contaminating cells from total splenic FRC samples, cells expressing one of the markers Lyve1, Hba-a1, Hba-a2, Ptprc, Tfrc, and Cldn5 were excluded from further analysis, reducing the cell number to 6856 cells. Similarly, in $\mathrm{EYFP}^{+}$samples, 276 cells expressing at least one of the markers Lyve1, Hba-a1, Hba-a2, or Krt18 were considered as non-FRC and excluded from further analysis. In addition, cells with exceedingly high or low number of detected genes or UMI counts (more than 2.5 median absolute deviations from the overall median, in either direction) were excluded, as were all cells with a large fraction of mitochondrial reads (more than 2.5 median absolute deviations above the median fraction). After these QC steps, 6227 cells were retained in total splenic FRC samples and 1318 cells were retained for $\mathrm{EYFP}^{+}$cell samples. Genes that were not assigned at least 1 read in at least two of these cells were filtered out, leaving 22,619 genes in total splenic FRC samples and 20,146 genes in EYFP ${ }^{+}$samples. Next, we used Seurat package in R (v2.3.0) ${ }^{71}$ to normalize the UMI counts, regress out the influence of the number of UMI counts per cell, and find highly variable genes. We also performed dimension reduction with PCA and t-SNE as well as cell clustering using Seurat for all datasets. For the $\mathrm{EYFP}^{+}$samples, all analyses were performed both for the full dataset and for the subsets with Ltbr-proficient and Ltbr-deficient $\mathrm{EYFP}^{+}$cells from adult spleens. In each of the datasets, one of the obtained clusters was inferred to contain both TRC and MRC cells and was further clustered into two subgroups using Seurat. Using canonical FRC markers and gene signatures from pop-RNA-seq, each cluster was assigned an informative label. The normalized expression values from Seurat were used to visualize expression levels of genes of interest across the inferred clusters as well as to find marker genes for each cluster, using the Wilcoxon test implemented in the Seurat package. In addition, for each cluster in the $\mathrm{EYFP}^{+}$samples, we used these values to calculate a signal-to-noise ratio (SNR) statistic for each gene, comparing the cells in the cluster to all cells outside the cluster, and thus generate a ranked list of genes for each cluster. These ranked lists were then supplied to GSEA-Preranked (v3.0) to investigate the enrichment of gene sets from the mSigDB (v5.2) collections C2, C5, and C7 downloaded from http://bioinf.wehi.edu. $\mathrm{au} /$ software/MSigDB/.

Differentiation trajectory analysis. In order to resolve differentiation trajectories we used the Monocle2 (v2.6.4) (PMID 28825705) package in R with normalized expression values from Seurat as input. The combination of the top 200 marker genes for each cluster (based on their $\operatorname{logFC}$ values) were selected for unsupervised ordering of the cells and the DDRTree algorithm implemented in Monocle2 was applied for trajectory reconstruction. To further resolve the differentiation towards TRC/MRC/FDC clusters, we reran the analysis on all cells that were aligned along the LT $\beta R$-dependent branch starting from the branching point.

Statistics. GraphPad Prism 7 was used for all statistical analyses. Differences with a $p$ value $<0.05$ were considered statistically significant.

Reporting summary. Further information on experimental design is available in the Nature Research Reporting Summary linked to this article.

\section{Data availability}

RNAseq and scRNA-seq data are deposited in the arrayexpress database (www.ebi.ac.uk/ arrayexpress) under accession numbers E-MTAB-7703, E-MTAB-7097, and E-MTAB7094. All other data are available from the authors upon reasonable requests.
Received: 5 October 2018 Accepted: 27 March 2019

Published online: 15 April 2019

\section{References}

1. Mebius, R. E. \& Kraal, G. Structure and function of the spleen. Nat. Rev. Immunol. 5, 606-616 (2005).

2. Brendolan, A., Rosado, M. M., Carsetti, R., Selleri, L. \& Dear, T. N. Development and function of the mammalian spleen. Bioessays 29, 166-177 (2007).

3. Waghorn, D. J. Overwhelming infection in asplenic patients: current best practice preventive measures are not being followed. J. Clin. Pathol. 54, 214-218 (2001).

4. Theilacker, C. et al. Overwhelming postsplenectomy infection: a prospective multicenter cohort study. Clin. Infect. Dis. 62, 871-878 (2016).

5. Geijtenbeek, T. B. et al. Marginal zone macrophages express a murine homologue of DC-SIGN that captures blood-borne antigens in vivo. Blood 100, 2908-2916 (2002).

6. Aichele, P. et al. Macrophages of the splenic marginal zone are essential for trapping of blood-borne particulate antigen but dispensable for induction of specific T cell responses. J. Immunol. 171, 1148-1155 (2003).

7. Ludewig, B. \& Cervantes-Barragan, L. CD169 macrophages take the bullet. Nat. Immunol. 13, 13-14 (2011)

8. Junt, T., Scandella, E. \& Ludewig, B. Form follows function: lymphoid tissue microarchitecture in antimicrobial immune defence. Nat. Rev. Immunol. 8 , 764-775 (2008).

9. den Haan, J. M., Mebius, R. E. \& Kraal, G. Stromal cells of the mouse spleen Front. Immunol. 3, 201 (2012).

10. Mueller, S. N. et al. Viral targeting of fibroblastic reticular cells contributes to immunosuppression and persistence during chronic infection. Proc. Natl Acad. Sci. USA 104, 15430-15435 (2007).

11. Scandella, E. et al. Restoration of lymphoid organ integrity through the interaction of lymphoid tissue-inducer cells with stroma of the $\mathrm{T}$ cell zone. Nat. Immunol. 9, 667-675 (2008).

12. Malhotra, D. et al. Transcriptional profiling of stroma from inflamed and resting lymph nodes defines immunological hallmarks. Nat. Immunol. 13, 499-510 (2012).

13. Chai, Q. et al. Maturation of lymph node fibroblastic reticular cells from myofibroblastic precursors is critical for antiviral immunity. Immunity 38, 1013-1024 (2013).

14. Astarita, J. L. et al. The CLEC-2-podoplanin axis controls the contractility of fibroblastic reticular cells and lymph node microarchitecture. Nat. Immunol. 16, 75-84 (2015).

15. Bekiaris, V. et al. Role of $\mathrm{CD} 30$ in $\mathrm{B} / \mathrm{T}$ segregation in the spleen. J. Immunol. 179, 7535-7543 (2007).

16. Cremasco, V. et al. B cell homeostasis and follicle confines are governed by fibroblastic reticular cells. Nat. Immunol. 15, 973-981 (2014).

17. Heesters, B. A., Myers, R. C. \& Carroll, M. C. Follicular dendritic cells: dynamic antigen libraries. Nat. Rev. Immunol. 14, 495-504 (2014).

18. Castagnaro, L. et al. Nkx2-5(+)islet1(+) mesenchymal precursors generate distinct spleen stromal cell subsets and participate in restoring stromal network integrity. Immunity 38, 782-791 (2013).

19. Magri, G. et al. Innate lymphoid cells integrate stromal and immunological signals to enhance antibody production by splenic marginal zone B cells. Nat. Immunol. 15, 354-364 (2014).

20. Rodda, L. B. et al. Single-cell RNA sequencing of lymph node stromal cells reveals niche-associated heterogeneity. Immunity 48, 1014-1028 e1016 (2018)

21. Buechler, M. B. \& Turley, S. J. A short field guide to fibroblast function in immunity. Semin. Immunol. 35, 48-58 (2018).

22. $\mathrm{Wu}, \mathrm{S}$. M. et al. Developmental origin of a bipotential myocardial and smooth muscle cell precursor in the mammalian heart. Cell 127, 1137-1150 (2006).

23. Novkovic, M. et al. Topological small-World Organization of the fibroblastic reticular cell network determines lymph node functionality. PLoS Biol. 14, e1002515 (2016).

24. Kumar Dubey, L., Karempudi, P., Luther, S. A., Ludewig, B. \& Harris, N. L. Interactions between fibroblastic reticular cells and B cells promote mesenteric lymph node lymphangiogenesis. Nat. Commun. 8, 367 (2017).

25. Gil-Cruz, C. et al. Fibroblastic reticular cells regulate intestinal inflammation via IL-15-mediated control of group 1 ILCs. Nat. Immunol. 17, 1388-1396 (2016).

26. Schonig, K., Schwenk, F., Rajewsky, K. \& Bujard, H. Stringent doxycycline dependent control of CRE recombinase in vivo. Nucleic Acids Res. 30, e134 (2002)

27. Onder, L. et al. Lymphatic endothelial cells control initiation of lymph node organogenesis. Immunity 47, 80-92 (2017).

28. Schaeuble, K. et al. Perivascular fibroblasts of the developing spleen act as LTalpha1beta2-dependent precursors of both T and B zone organizer cells. Cell Rep. 21, 2500-2514 (2017). 
29. Mendez-Ferrer, S. et al. Mesenchymal and haematopoietic stem cells form a unique bone marrow niche. Nature 466, 829-834 (2010).

30. Morrison, S. J. \& Scadden, D. T. The bone marrow niche for haematopoietic stem cells. Nature 505, 327-334 (2014).

31. Morikawa, S. et al. Prospective identification, isolation, and systemic transplantation of multipotent mesenchymal stem cells in murine bone marrow. J. Exp. Med. 206, 2483-2496 (2009).

32. Livet, J. et al. Transgenic strategies for combinatorial expression of fluorescent proteins in the nervous system. Nature 450, 56-62 (2007).

33. Qiu, X. et al. Reversed graph embedding resolves complex single-cell trajectories. Nat. Methods 14, 979-982 (2017).

34. El Agha, E. et al. Mesenchymal stem cells in fibrotic disease. Cell Stem Cell 21, 166-177 (2017).

35. Armulik, A., Genove, G. \& Betsholtz, C. Pericytes: developmental, physiological, and pathological perspectives, problems, and promises. Dev. Cell 21, 193-215 (2011).

36. Benezech, C. et al. Lymphotoxin-beta receptor signaling through NFkappaB2-RelB pathway reprograms adipocyte precursors as lymph node stromal cells. Immunity 37, 721-734 (2012).

37. van de Pavert, S. A. et al. Chemokine CXCL13 is essential for lymph node initiation and is induced by retinoic acid and neuronal stimulation. Nat. Immunol. 10, 1193-1199 (2009).

38. Katakai, T. Marginal reticular cells: a stromal subset directly descended from the lymphoid tissue organizer. Front. Immunol. 3, 200 (2012).

39. Jarjour, M. et al. Fate mapping reveals origin and dynamics of lymph node follicular dendritic cells. J. Exp. Med. 211, 1109-1122 (2014).

40. Krautler, N. J. et al. Follicular dendritic cells emerge from ubiquitous perivascular precursors. Cell 150, 194-206 (2012).

41. Sitnik, K. M. et al. Context-dependent development of lymphoid stroma from adult CD34(+) adventitial progenitors. Cell Rep. 14, 2375-2388 (2016).

42. Tan, J. K. \& Watanabe, T. Stromal cell subsets directing neonatal spleen regeneration. Sci. Rep. 7, 40401 (2017).

43. de Souza, L. E., Malta, T. M., Kashima Haddad, S. \& Covas, D. T. Mesenchymal stem cells and pericytes: to what extent are they related? Stem Cells Dev. 25, 1843-1852 (2016).

44. Crisan, M. et al. A perivascular origin for mesenchymal stem cells in multiple human organs. Cell Stem Cell 3, 301-313 (2008).

45. Kramann, R. et al. Perivascular Gli1+ progenitors are key contributors to injury-induced organ fibrosis. Cell Stem Cell 16, 51-66 (2015).

46. Guimaraes-Camboa, N. et al. Pericytes of multiple organs do not behave as mesenchymal stem cells in vivo. Cell Stem Cell 20, 345-359 e345 (2017).

47. Cooper, M. D. \& Alder, M. N. The evolution of adaptive immune systems. Cell 124, 815-822 (2006).

48. Boehm, T., Hess, I. \& Swann, J. B. Evolution of lymphoid tissues. Trends Immunol. 33, 315-321 (2012).

49. Pitchappan, R. Review on the phylogeny of splenic structure and function. Dev. Comp. Immunol. 4, 395-416 (1980).

50. Cooper, M. D., Peterson, R. D. \& Good, R. A. Delineation of the thymic and bursal lymphoid systems in the chicken. Nature 205, 143-146 (1965).

51. Jeurissen, S. H., Claassen, E. \& Janse, E. M. Histological and functional differentiation of non-lymphoid cells in the chicken spleen. Immunology 77 , 75-80 (1992).

52. Matzinger, P. Essay 1: the danger model in its historical context. Scand. J. Immunol. 54, 4-9 (2001).

53. Lane, P. J. et al. Lymphoid tissue inducer cells: bridges between the ancient innate and the modern adaptive immune systems. Mucosal Immunol. 2, 472-477 (2009).

54. Treml, L. S., Crowley, J. E. \& Cancro, M. P. BLyS receptor signatures resolve homeostatically independent compartments among naive and antigenexperienced B cells. Semin. Immunol. 18, 297-304 (2006).

55. Boyman, O., Letourneau, S., Krieg, C. \& Sprent, J. Homeostatic proliferation and survival of naive and memory T cells. Eur. J. Immunol. 39, 2088-2094 (2009).

56. Tumanov, A. V. et al. Cellular source and molecular form of TNF specify its distinct functions in organization of secondary lymphoid organs. Blood 116, 3456-3464 (2010).

57. Glenney, G. W. \& Wiens, G. D. Early diversification of the TNF superfamily in teleosts: genomic characterization and expression analysis. J. Immunol. 178, 7955-7973 (2007).

58. Sparwasser, T. \& Eberl, G. BAC to immunology-bacterial artificial chromosome-mediated transgenesis for targeting of immune cells Immunology 121, 308-313 (2007).

59. Miyazaki, S., Miyazaki, T., Tashiro, F., Yamato, E. \& Miyazaki, J. Development of a single-cassette system for spatiotemporal gene regulation in mice. Biochem. Biophys. Res. Commun. 338, 1083-1088 (2005).

60. Wimmer, N. et al. Lymphotoxin beta receptor activation on macrophages induces cross-tolerance to TLR4 and TLR9 ligands. J. Immunol. 188, 3426-3433 (2012).
61. Bolger, A. M., Lohse, M. \& Usadel, B. Trimmomatic: a flexible trimmer for Illumina sequence data. Bioinformatics 30, 2114-2120 (2014).

62. Dodt, M., Roehr, J. T., Ahmed, R. \& Dieterich, C. FLEXBAR-flexible barcode and adapter processing for next-generation sequencing platforms. Biology $\mathbf{1}$, 895-905 (2012)

63. Dobin, A. et al. STAR: ultrafast universal RNA-seq aligner. Bioinformatics 29, 15-21 (2013).

64. Patro, R., Duggal, G., Love, M. I., Irizarry, R. A. \& Kingsford, C. Salmon provides fast and bias-aware quantification of transcript expression. Nat. Methods 14, 417-419 (2017).

65. Soneson, C., Love, M. I. \& Robinson, M. D. Differential analyses for RNAseq: transcript-level estimates improve gene-level inferences. F1000Res 4, 1521 (2015).

66. Robinson, M. D., McCarthy, D. J. \& Smyth, G. K. edgeR: a Bioconductor package for differential expression analysis of digital gene expression data. Bioinformatics 26, 139-140 (2010).

67. Ritchie, M. E. et al. limma powers differential expression analyses for RNA-sequencing and microarray studies. Nucleic Acids Res. 43, e47 (2015).

68. Macosko, E. Z. et al. Highly parallel genome-wide expression profiling of individual cells using nanoliter droplets. Cell 161, 1202-1214 (2015).

69. Zheng, G. X. et al. Massively parallel digital transcriptional profiling of single cells. Nat. Commun. 8, 14049 (2017).

70. McCarthy, D. J., Campbell, K. R., Lun, A. T. \& Wills, Q. F. Scater: preprocessing, quality control, normalization and visualization of single-cell RNA-seq data in R. Bioinformatics 33, 1179-1186 (2017)

71. Butler, A., Hoffman, P., Smibert, P., Papalexi, E. \& Satija, R. Integrating singlecell transcriptomic data across different conditions, technologies, and species. Nat. Biotechnol. 36, 411-420 (2018).

\section{Acknowledgements}

This study received financial support from the Swiss National Science Foundation (Grants 166500 and 159188 to B.L.), the Human Frontiers Science Program (RGP0034/ 206), and the Deutsche Forschungsgemeinschaft (HE3116/9-1 to T.H. and B.L.). The funders had no role in study design, data collection and analysis, decision to publish, or preparation of the paper.

\section{Author contributions}

B.L. designed the study, discussed the data and wrote the paper; H.-W.C. performed the experiments, analyzed the data and wrote the paper; L.O., M.N., C.S., N.P., M.L. and E.S performed the experiments, analyzed and discussed the data; J.M., A.T., U.S. and K.P. provided the reagents; T.R. produced the transgenic mice and discussed the data, M.R. and T.H. supported the study design and discussed the data.

\section{Additional information}

Supplementary Information accompanies this paper at https://doi.org/10.1038/s41467019-09728-3.

Competing interests: The authors declare no competing interests.

Reprints and permission information is available online at http://npg.nature.com/ reprintsandpermissions/

Journal peer review information: Nature Communications thanks Yang-Xin Fu and the other anonymous reviewer(s) for their contribution to the peer review of this work. Peer reviewer reports are available.

Publisher's note: Springer Nature remains neutral with regard to jurisdictional claims in published maps and institutional affiliations.

Open Access This article is licensed under a Creative Commons Attribution 4.0 International License, which permits use, sharing, adaptation, distribution and reproduction in any medium or format, as long as you give appropriate credit to the original author(s) and the source, provide a link to the Creative Commons license, and indicate if changes were made. The images or other third party material in this article are included in the article's Creative Commons license, unless indicated otherwise in a credit line to the material. If material is not included in the article's Creative Commons license and your intended use is not permitted by statutory regulation or exceeds the permitted use, you will need to obtain permission directly from the copyright holder. To view a copy of this license, visit http://creativecommons.org/ licenses/by/4.0/.

(c) The Author(s) 2019 